\title{
Molecularly imprinted polymers - towards electrochemical sensors and electronic tongues
}

\author{
Anna Herrera-Chacón ${ }^{1} \cdot$ Xavier Cetó $^{1} \cdot$ Manel del Valle ${ }^{1}$ (B) \\ Received: 18 February 2021 / Revised: 18 March 2021 / Accepted: 24 March 2021 / Published online: 30 April 2021 \\ (C) Springer-Verlag GmbH Germany, part of Springer Nature 2021
}

\begin{abstract}
Molecularly imprinted polymers (MIPs) are artificially synthesized materials to mimic the molecular recognition process of biological macromolecules such as substrate-enzyme or antigen-antibody. The combination of these biomimetic materials with electrochemical techniques has allowed the development of advanced sensing devices, which significantly improve the performance of bare or catalyst-modified sensors, being able to unleash new applications. However, despite the high selectivity that MIPs exhibit, those can still show some cross-response towards other compounds, especially with chemically analogous (bio)molecules. Thus, the combination of MIPs with chemometric methods opens the room for the development of what could be considered a new type of electronic tongues, i.e. sensor array systems, based on its usage. In this direction, this review provides an overview of the more common synthetic approaches, as well as the strategies that can be used to achieve the integration of MIPs and electrochemical sensors, followed by some recent examples over different areas in order to illustrate the potential of such combination in very diverse applications.
\end{abstract}

Keywords Molecularly imprinted polymers (MIPs) · Voltammetric sensors · Electronic tongue $\cdot$ Molecular imprinting · Polymerization $\cdot$ Immobilization

\section{Introduction}

Over the last decades, there has been an exponential increase in the demand for the identification, classification or quantification of chemical and/or biological species in more or less complex matrices. Such increase is not limited to a particular field, but a general trend of a more knowledgeable society that demands, from the stakeholders and policymakers, a better understanding, monitoring and control of the composition of daily life products. Some examples of these increases can be found in the environmental field, where the number of emerging contaminants is growing continuously (with over 100,000 chemicals registered) $[1,2]$; food control, where analytic

Published in the topical collection Analytical Applications of Biomimetic Recognition Elements with guest editors Maria C. Moreno-Bondi and Elena Benito-Peña.

Manel del Valle

manel.delvalle@uab.cat

1 Sensors and Biosensors Group, Department of Chemistry, Universitat Autònoma de Barcelona, Edifici Cn, 08193 Bellaterra, Barcelona, Spain methods have turned into a key element to improve its quality and to allow its traceability [3]; clinical diagnostics, where the identification of key biomarkers and their normal ranges allows the detection of several pathologies [4]; etc.

Beyond the challenge that the analysis of such compounds can represent by itself, there is also a demand for such methods to be economic, fast-response, simple, portable and low-energy consumption. In this direction, electrochemical sensors offer fast and accurate information in a cost-effective manner. Besides, their high sensitivity, wide linear range, minimal power requirement, potential for miniaturization and portability, as well as ease of operation, are some of their several advantages [5]. For these reasons, among the incorporation of durable and reusable sensors, the field of sensing and biosensing is gaining popularity among the scientific community and is one of the most growing fields in recent publications.

In order to improve the performance and selectivity of sensors, many different materials and (bio)compounds have been used when developing electrochemical sensors [6, 7]. On the one side, there is the usage of (bio)catalysts (such as enzymes) that specifically catalyse the conversion of the target analyte to another product more easily detectable, or electrocatalysts that act as mediators for a more efficient electron exchange and/or 
faster reaction rate, which results in, e.g. a lower oxidation/ reduction potential and/or increase in the sensitivity [8]. In this sense, the progress in nanoscience has led to a range of new nano-structured materials that have emerged as an alternative to the respective bulk materials given its higher surface/mass ratio and improved electrochemical properties [9]. On the other side, there is the usage of (bio)chemical recognition elements that do not affect the electrochemical responses directly, but enhance the selectivity of the developed sensors by specifically binding to the desired analyte (e.g. affinity biosensors, which include immunosensors and genosensors) [10]. Despite the high performance of such type of biosensors, those present some drawbacks such as its cost, long-term stability or instability out of the physiological conditions, between others. In this direction, to spawn new application fields, there is a necessity to obtain synthetic biomimetic recognition elements that may substitute natural analogues such as antibodies or enzymes. Advantages of working with these artificial receptors are not only because of its affordability but also due to the possibility to work in a wide range of conditions ( $\mathrm{pH}$, temperature, solvents, etc.) or out of strict physiological conditions.

In this context, several synthetic (bio)molecules have been evaluated from the principles of host-guest chemistry, including the use of aptamers (aptasensors), molecularly imprinted polymers (MIPs), crown ethers or cyclodextrins; more recently, the use of metal-organic frameworks (MOFs) or covalent organic frameworks (COFs) has also attracted significant attention as artificial receptors. However, the poor chemical stability in aqueous solutions and low electrical conductivity of the latter hinders their use in electrochemical systems [11]. Among the above list, aptamers and MIPs have clearly demonstrated a potential and performance above the rest, but with MIPs still being a cheaper and more versatile option in terms of operation conditions given its higher stability.

Among biomimetic artificial receptors, MIPs represent a promising alternative due to its simple synthesis, comparable performance to affinity bioreceptors, tunability, high stability and low-cost. These polymers are synthesised based on template-induced formation of complimentary recognition cavities, which are thus made specific in shape, size and functionality against a target chemical or biological molecule (the template). Indeed, the recognition of the template by the MIP has been compared to the Fischer's lock and key analogy used to describe the preferential interaction between an enzyme and its substrate [12]. MIPs synthesis commonly starts by mixing the template with one or more functional monomer(s) in an appropriate solvent, which allows the formation and solubilisation of the pre-polymerization complex (based on complementary forces between chemical groups of both molecules). After that, there is usually the addition of a crosslinker and a radical initiator, commonly under free oxygen conditions, mechanism that knits the polymer around the previously mentioned complex. Upon synthesis of the polymer, the next step is the removal of the template molecule, in order to yield a material able to selectively rebind to the target analyte with high specificity even in the presence of structurally analogue interfering compounds. Non-imprinted polymers (NIPs) are synthesised in the same manner, but without the template presence for comparison purposes. As schematized in Fig. 1, in the case of MIP synthesis, the monomer(s) are first spontaneously arranged around the template to obtain a tailor-made cavity complementary to it. If we consider the NIP synthesis, there is no arrangement process due to the absence of the template; producing a polymer without imprinting (against the template molecule) and therefore a less functional cavity. Reason for which the NIP polymer is used as a control of the synthesis by itself.

Finally, in order to be used for electrochemical sensing, as the synthesis of these polymers provides plastic insulator materials, the proper integration into chemical sensors (where the transducer will convert the binding event into an analytical signal) is required. This is the reason why optical applications are more common when employing MIPs. Despite their poor electrical properties, their incorporation into chemical sensors clearly improves the selectivity and the sensitivity of the latter, as MIPs allow the extraction and pre-concentration of the target analyte from sample complex matrices, therefore permitting an enrichment and posterior stripping methodology.

As these materials gain attention, new synthetic routes, polymerizations and immobilizations are being explored in order to improve the overall performance of electrochemical sensors. In this direction, this manuscript aims to present and critically review the more common approaches for the synthesis of MIPs as biomimetic synthetic receptors, and their incorporation into electrochemical sensors. Finally, some of the most relevant applications recently reported in the literature over different scenarios will also be reviewed in order to illustrate the potential of MIPs in combination with electrochemical sensors.

\section{Synthesis}

Molecular imprinting includes the design and synthesis of the molecular recognition polymers and comprises a plethora of possibilities. The field started in the 1930s when Polyakov reported an unusual selective behaviour of some silica particles depending on the different additives and solvents employed during its preparation [13]. This was in the same decade that the idea of the antibody recognition was under debate by Bernil [14] and Pauling [15]. Some years afterwards, Dickey [16], in the same direction that Polyakov did earlier, published about the precipitation of highly selective silica gel particles towards dye methyl orange with other different dyes present. But it was not until 1952 that this idea was Nobel laureated when Martin and Synge presented their study 
a
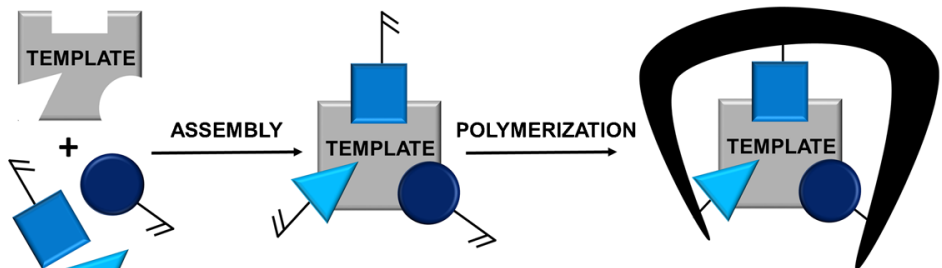

EXTRACTION

REBINDING

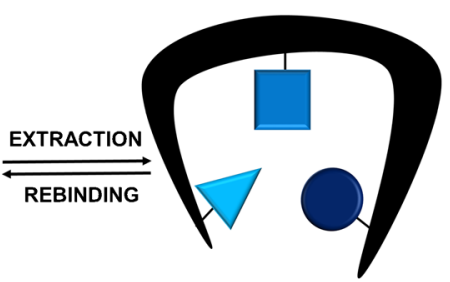

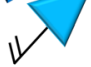

b
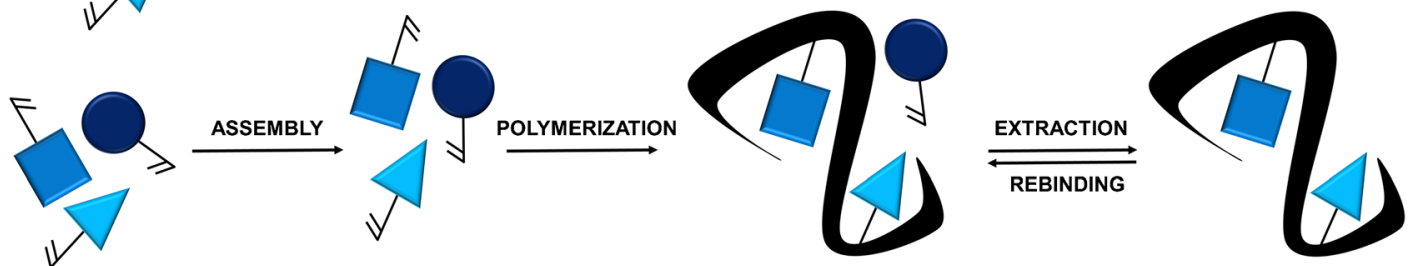

Fig. 1 Schematic representation of the synthesis of biomimetic artificial receptors: (A) molecularly imprinted polymer (MIP) and (B) non-imprinted polymer (NIP)

describing the induction of selective footprints using methyl orange as an indicator ligand during the separation of amino acid derivatives [17]. The former settled the basis for what Klotz [18] and Wulff [19] described as molecular imprinting when reporting the synthesis of a non-siliceous cross-linked polymer around a template molecule, and Sagiv [20] about the concept of silica imprinting. However, it was not until the 1990s that the interest on exploiting MIPs started to grow exponentially, developing MIPs towards a diverse range of templates such as ions, molecules, macromolecules, viruses, micro-organisms or nanoparticles, among others.

Prior to moving forward to the synthesis of MIPs, special mention has to be made to the NIP and its importance. For binding comparative purposes, a NIP should be synthesised in parallel. The aim is to demonstrate that there is actually an imprinting effect on the synthesized polymer. In this manner, by using the NIP as a benchmark for our MIP, it is possible to evaluate the imprinting factor (IF $=K_{\mathrm{MIP}} / K_{\mathrm{NIP}}$, where $K$ is the affinity constant or the sensor's response), which is a measure of the strength of the interaction of the imprinted polymer towards the template molecule. Therefore, the best MIP is the one that has the largest IF value, confirming a good imprinting, while values close to 1 indicate that the imprinting effect is almost negligible, which means that the interaction between the MIP and the template is rather unspecific, more related to surface interactions than to any generated cavities.

As previously mentioned, the imprinting technique is based on the formation and arrangement of the pre-polymerization complex between the template and the functional monomer(s), followed by the synthesis of the polymer matrix on its surroundings, and finally, the template removal [21, 22]. Precisely, the main difference among the different approaches to perform the synthesis is the nature of the prepolymerization binding and how the template molecule is finally removed (Fig. 2). In this direction, over the next subsections, the more common approaches for the synthesis of MIPs will be presented and their main advantages/disadvantages will be discussed, taking into account its suitability to be later used for the development of electrochemical sensors.

\section{Covalent imprinting}

Covalent imprinting method was developed and first reported by Wulff and Sarhan [19, 23], for the imprinting of D-glyceric acid (the template) using molecules with boronic acid and amine-based functionalities for the steric arrangement and polymerizable vinyl groups combined with divinylbenzene as cross-linker. Molecular imprinting via covalent forces was the first method reported for the design of artificial receptors. This method is highly suitable when a great level of precision on the specificity of the imprinting is desired due to the high stability of the template-monomer(s) complex, which arises from the strong bonds formed between them, and that theoretically leads to highly specific and high-affinity MIPs. However, what is its main advantage becomes also its major drawback given the difficulty to remove the template once the MIP is synthesized. This is evidenced in the poor recovery of the template after the polymerization, but also in the increased difficulty in the rebinding/removal of the template when used as a MIP, which leads to slower kinetics.

To proceed with this approach, the template molecule requires having at least one functional group suitable to be derivatized; common examples found in the literature include alcohols, aldehydes, ketones, amines or carboxylic acids. After creating the tailor-made cavities, the template needs to be removed by chemically cleaving the bonds with the synthesized MIP. This can be achieved by, e.g. acidic hydrolysis or reductive cleavage of the ester bonds with concentrated acids or bases, or through the application of high pressure or supercritical $\mathrm{CO}_{2}$, among other ways. Thus, other drawbacks of this synthetic approach are: (1) the template modification implies an extra step, and (2) the limited number of species that may form a covalent bond with reversible properties (the list includes carboxylic and boronic esters, ketals, disulphide 
Fig. 2 Diagram of the main imprinting methods: a Covalent and semi-covalent imprinting, $\mathbf{b}$ metal-ion exchange imprinting and $\mathbf{c}$ non-covalent imprinting

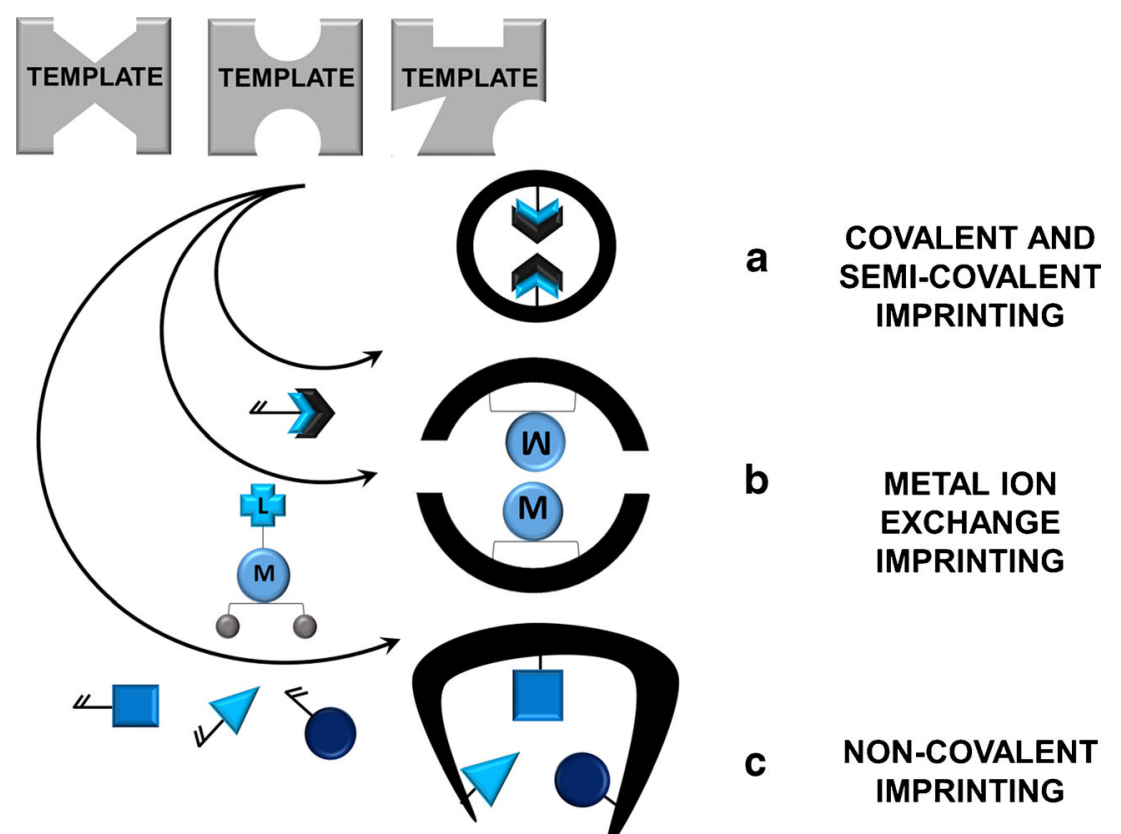

bonds, acetals and imines), which translates into a limitation of the species that can be used as a template and, consequently, for which a MIP can be synthesized by this strategy.

\section{Non-covalent imprinting}

Non-covalent imprinting is by far the most widespread methodology. Mosbach's group promoted and disseminated this approach in the 1980s decade [24-26], causing a great popularization of the imprinting technique and an exponential growth in publications over the 1990s and 2000s [27] that seems it has not stopped yet. The nature of the prearrangement in non-covalent imprinting (with a wider range of chemical functionalities and a relatively simpler synthesis process) became a milestone that opened a full variety of uses and applications over several scientific areas. The types of interactions that are predominant for the formation of the self-assembled pre-polymerization complex in this approach are the Van der Waals forces, dipole-dipole, charge-dipole, cation- $\pi, \pi$-stacking, hydrogen bonding or electrostatic (charge-charge), between others. Whereas for the synthesis of the MIP, functional monounsaturated monomers such as vinylic, acrylic or methacrylic monomer are used; together with a high amount of cross-linker from the same nature (vinylic, acrylic or methacrylic). The mechanism of choice is usually free radical polymerization (thermally or UV induced) which provides the final organic network material.

The main advantages of non-covalent imprinting are the limitless type of templates that could be used, its ease of preparation, the faster and more efficient template removal and, significantly, the more favourable rebinding kinetics. However, the specificity of the binding sites is clearly compromised due to the heterogeneity from the different pre-polymerization complexes formed, the direct competition from the solvent, the cross-linker (that is added in high proportion in order to obtain a polymer with more rigidity) or the underestimation of the chaos generated by polymerization process that is often promoted by thermal-induced polymerization process, the so-called free radical polymerization (FRP).

\section{Semi-covalent imprinting}

The semi-covalent approach seeks to combine the advantages of both the covalent and the non-covalent synthesis, while counterbalancing their disadvantages. This approach can be attributed to Wülff [28], but it was not until a few years later when it was formally reported with the use of "sacrificial spacer" by Whitcombe et al. [29]. The main idea is to create a MIP based on covalent imprinting (pre-polymerization complex with covalent bonds), and after the polymerization step, the covalent features involved are replaced by non-covalent functionalities so that the rebinding of the template is based on non-covalent interactions. This mixed process thus benefits of the lower non-specific interactions and highly precise cavities of covalent imprinting, and of the more favourable template rebinding process of non-covalent imprinting (the rebind is only affected by diffusion parameters, without counting any other kinetic parameter than diffusion).

However, crowding and steric hindrance between the MIP and the template may arise due to the change in functionalities, which may cause a loose in the polymer binding capacity, hampering the imprinting. In order to overcome such drawback, a linker group (the so-called sacrificial spacer) may be 
used to connect the template and the monomer(s), which is lost during the template removal. In this manner, the sacrificial spacer serves, first, to covalently attach the template and the functional monomer(s) during the pre-polymerization, while at the same time serves to prevent steric effects upon template removal. Some examples of sacrificial spacer groups that can be found in the literature include the use of carbonate ester, carbamate, urea, salicylamide or silyl ether. Nevertheless, the use of sacrificial spacers reduces the list of possibilities of tailor-made cavities by imprinting technique, adds an extra coupling step that needs to be optimised and provides a lesseffective template recognition if the necessary attraction dipoles are not strong enough.

\section{Metal-ion exchange imprinting}

Similar to semi-covalent imprinting method, which arose as an alternative that sought to improve the performance of existing methodologies, metal-ion exchange imprinting aims to take advantage of metal ions to create ionic bonds (which are stronger than hydrogen bonds or electrostatic interactions) that facilitate the interaction between the monomer and the template molecule, while also combine the benefits of covalent and non-covalent imprinting. That is, metal ions are used as an assembly pivot for the formation of the pre-polymerization complex. The metal ion may form a complex between the template with monomer(s) that form coordinative bonds with metals, or by using ionophores for the imprinting. Interaction of the metal ions and stability of the complexes formed can be tuned by varying the metal, its oxidation state and/or the ligand characteristics. Moreover, apart from being used as a monomer through the integration of the accompanying ligand (e.g. porphyrins) into the polymeric material, they can also act as template for the imprinting of metal ions.

This approach was first proposed by Fujii et al. for the imprinting of a polymer able to discriminate between the stereoisomers of an amino acid [30], demonstrating the huge potential of the obtained MIP and the approach. Despite its evident potential, is clear that this approach is limited to strategies containing complex-forming groups with metals.

\section{In situ electropolymerized MIP sensors}

The first electropolymerized MIP-based sensor was reported in 1999 by Malitesta et al. for the detection of glucose by quartz crystal microbalance (QCM) [31]. The MIP was obtained from a solution that contained o-phenylenediamine (oPD) as the electropolymerizable monomer, the template and a supporting electrolyte. In the procedure, the authors achieved both the synthesis and immobilization of the MIP onto the electrode surface at the same time. Furthermore, a cross-linking co-monomer or different nanomaterials may be added during the polymerization, achieving their incorporation into the polymeric matrix. Lastly, the removal of the template could be done by, e.g. overoxidation of the polymer or the template itself, a step that is extremely fast in comparison with other approaches.

As already commented, a main asset of this approach is that it consists of a one-step electro-synthesis, which allows the integration of the material onto the electrode surface with good adherence, and that the thickness of the MIP layer can be tuned by changing the total charge flown at the electrode, the polymerization media or the electrolyte nature. On the other hand, the main drawbacks are related to the more limited choice of monomer(s) and the interactions that those might have with the template, and that the template should be stable during the synthesis step in the potential window used for the electropolymerization to ensure that precise and specific cavities are generated. No only this, but also the other components that may be added into the mixture need to not have functional groups able to polymerize to avoid creating heterogeneous binding sites.

Despite not being considered yet a separate category for the imprinting technique [32], the use of electropolymerized MIPs in (bio)sensing has skyrocketed over the last years. Recently, published works seem to favour in this approach due to its advantages, such as its simplicity or its integration ability. Some examples include the use of polymerizable functional monomer(s) such as o-phenylenediamine (oPD), aniline, thiophene or thiophene derivatives (Th), chitosan $(\mathrm{Ch})$, acrylamide and the most widely used pyrrole (Py), alone or in derivatized forms.

\section{Strategies to improve molecular imprinting}

\section{Computational approaches for the design of MIPs}

Although it does not represent a synthetic route itself, and its usage precedes the actual synthesis of the imprinted polymer, we consider that special mention is deserved to the use of computational methods in the design of MIPs, which may facilitate further improvement of the high affinity of such polymers through rational design protocols [33-36], with evident reduction of experimental effort. Computational methods for receptor design appeared in the late 1990s, but it was not until 2001 when Piletsky et al. demonstrated that good predictions could be obtained, avoiding running large sets of experiments that these methods were widely acknowledged [37]. In this manner, screening of the functional monomer, selection of the cross-linker and the solvent, optimisation of monomer(s)-template ratio or selectivity analysis are computationally simulated by molecular modelling and computational design. To this aim, several approaches have been reported in the literature, including molecular mechanics/ molecular dynamics or one of the broad quantum 
mechanics-based techniques (semi-empirical, ab initio or density functional theory (DFT)). Alternatively, chemometric methods have also been used for the optimization of synthesis parameters $[38,39]$. The advantages derived from the use of such methods are the preparation of high-affinity polymers with control over their binding strength on the one side, while also allowing for a significant reduction in time and resources that would be required with empirical screening methods on the other side. Moreover, these methods contribute to the greater understanding of monomer-template complexation and the impact of polymerisation on the structure of the binding site, unveiling the production of more advanced materials (e.g. responsive materials).

\section{Dummy MIPs}

Although one of the advantages of MIPs is the possibility to synthetize highly selective receptors for almost any (bio)compound, there is certain situations in which this is not as straightforward. Namely, when the target template (i) is unstable under the conditions required to carry out the polymerization or its poor solubility hinders the imprinting, (ii) it is considered a hazard or safety concerns arise from its manipulation, (iii) is very expensive or (iv) to solve problem of template leakage. In such scenarios, a structural analogue might be used as the template to carry out the imprinting instead of the target analyte; an approach that is known as dummy molecular imprinting or simply referring to the synthetized polymer as dummy MIP (DMIP). This was first reported by Andersson et al. [40], although the term was not coined until a few years later. Examples of scenarios in which DMIPs can be considered the more attractive or effective approach involve the synthesis of MIPs towards compounds such as TNT due to being an explosive [41] or bisphenol A (BPA) due to its leakage [42].

\section{Polymerization}

The polymerization is a key part of the MIP synthesis process as the final polymer form changes the whole functionality and affects its main purpose, the imprinting. For example, a fast uncontrolled polymerization reaction might generate a polymer with non-accessible cavities or generate a non-rigid polymer that may become useless depending on its intended application. In this sense, a plethora of polymerization approaches can be used, from which over the next subsections we will focus on the more common polymerization types and methods. Moreover, the polymerization step can be further tuned by changing the ratio of the solvent over the other reagents, the ratio of the monomers and the template, the initiation method or source, the surface or place where it occurs, etc. Besides, the polymer chains can be incorporated to several surfaces to form different architectures such as core-shells, layers or beads.

\section{Type of polymerization}

Among the different types of polymerization, MIPs are generally produced by free radical polymerization (FRP), controlled radical polymerization (CRP), photopolymerization or electropolymerization [43].

From those, FRP is the most widely used strategy. The reaction is conducted under mild conditions, either in bulk or in solution, and it may be initiated by heating or UV-vis irradiation. It can be used with several monomers, and from the preparation point of view, it provides a simple and rapid approach. Some disadvantages are that it does not allow control over the size of the macromolecules, the irreversible termination reaction and the possibility of uncontrolled atom transfers.

In this direction, there are other variants that could help to control the size, shape and molecular weight distribution such as CRP or living radical polymerization (LRP). CRP is mainly used in through activation-deactivation cycles, which help to control the polymer growth and to obtain polymeric chains of similar length. CRP can be used for several types of chemical species, and includes several strategies such as atom transfer radical polymerization (ATRP), nitroxide-mediated polymerization (NMP), iniferter-mediated polymerization or reversible additionfragmentation chain transfer polymerization (RAFT). As already mentioned, the main advantage of CRP methods is that it yields monodisperse polymer particles with uniform morphology, e.g. as it happens with dendrimer structures.

Photopolymerization is mainly employed to obtain thin films with controlled thickness requirements, often in the nano-scale level. Its main advantages are the tight control on the film thickness. However, given the low penetration of the UV radiation, there is a limit in the thickness or size of the polymer. Another limitation is that it may not be suitable for all molecules as it might be the case where the template may degrade once exposed to UV radiation.

Lastly, electropolymerization approach is mainly used to obtain films directly onto the electrode surface, as already described in the "In situ electropolymerized MIP sensors" section. For obvious reasons, this option has been mostly employed in the field of electrochemical sensors. Its main advantages are the relative simplicity of the polymerization process compared to the previous methods, the high reproducibility and control over the polymerization, or the compatibility with aqueous media.

\section{Method of polymerization}

Checking MIP progress, by the 1990s decade, the imprinting technique gained popularity by offering bulk materials with 
precisely tailor-made cavities. However, the created porous were not always as desired, and the size of the obtained particles was not homogeneous enough even after grinding the monolith; all this produced a limited material with template leakage and poor posterior rebinding. Especially in the electrochemical field, this material was inadequate due to its difficult integration into electrochemical sensors, particularly in the commonly preferred form: a thin layer incorporating this biomimetic polymer to provide a better and enhanced transduction thanks to improved diffusion.

Due to the disadvantages that the monolith synthesis presented, several alternative approaches were developed in the following years, advancing in new synthesis and polymerization strategies that facilitate the incorporation of these artificial receptors into new sensing platforms. The revolution became when MIPs were synthesized as nanoparticles (NPs), offering a higher-surface-to-area ratio and improving their binding kinetics, commonly through precipitation polymerization.

In this regard, MIPs prepared by FRP, CRP or LRP can be prepared by bulk, precipitation, emulsion, core-shell, suspension, multi-step polymerization, etc., which choice will determine how polymer particles are obtained. Over the next subsections, those will be briefly presented, while Table 1 summarizes the different polymerization strategies.

\section{Bulk or monolith}

Bulk imprinted polymers are obtained with a quick synthetic approach that yields a monolith. This monolith material is usually grinded and meshed to obtain polymer fractions with a controllable particle size. Due to its simplicity, robustness and the possibility to work in a wide range of solvents, pressure and temperature, this approach is widely used in chromatographic applications, e.g. for use as column's stationary phases in high-performance liquid chromatography (HPLC) or in solid-phase extraction cartridges (SPE). The main drawback derives from the monolith treatment. On the one side, those steps are time consuming and there is a loss of polymer during the grinding and sieving until obtaining a controlled particle size. On the other side, given the possible damage to its structure during the mechanical grinding, there is a risk of altering the imprinting. Moreover, the pores are not as accessible as in other approaches, which also might hinder the mass transport and diffusion. For this reason, the template removal is more problematic, which is reflected in the very poor recovery of the template after the polymerization. An example of this synthesis can be found in the work of Urraca et al. for the non-covalent imprinting of zearalenone [44], an estrogenic mycotoxin, or for the synthesis of water-compatible MIP for fluoroquinolone antibiotics using enrofloxacin (a fluoroquinolone) as the template [45].

\section{Precipitation polymerization}

Precipitation polymerization is a superb approach to obtain uniform and spherical sub-micrometre particles with the proper imprinting properties. Different ratios of reagents can be used from a highly cross-linked to an excess of solvent mixture, which converts this approach in one of the more versatiles, and consequently, one of the most commonly used. The polymer particles grow in solution until they reach the critical size, at which moment they precipitate until the initiator is consumed or deactivated from its source. The main advantages are that there is no necessity to modify the template and monomer(s) complex, the obtained particles will have a higher surface-to-volume ratio compared with bulk polymerization and the template removal will be easier (consequently, not requiring strong treatments). However, its main drawback is that the diluted media leads to heterogeneous cavities based in several low-affinity interactions that might affect its selectivity as MIP. In this manner, Rebelo et al. combined computationally studies with precipitation polymerization to develop a voltammetric sensor for furazolidone antibiotic [46]. Similarly, Gholivand et al. prepared a MIP towards methocarbamol, which was later used as selective adsorbent to develop a molecularly imprinted solid-phase extraction, either with voltammetric or chromatographic detection [47]. Using the same approach, another voltammetric MIP-based sensor towards histamine was prepared to detect and quantify histamine in wine samples (Fig. 3) [48]. Another interesting

Table 1 Summary of the main advantages and disadvantages of the more common polymerization methods

\begin{tabular}{|c|c|c|c|}
\hline $\begin{array}{l}\text { Polymerization } \\
\text { method }\end{array}$ & Advantages & Disadvantages & $\begin{array}{l}\text { Example of reported } \\
\text { (bio)sensing applications }\end{array}$ \\
\hline Bulk & Simple and cost-effective & $\begin{array}{l}\text { Harsher template removal and heterogeneity in } \\
\text { particle size distribution and/or cavities }\end{array}$ & {$[44,45]$} \\
\hline Precipitation & $\begin{array}{l}\text { Quick synthesis and easier template removal } \\
\text { with high yields }\end{array}$ & $\begin{array}{l}\text { Diluted reagents media producing heterogeneous } \\
\text { cavities }\end{array}$ & [46-49] \\
\hline Emulsion & Small nanoMIPs & $\begin{array}{l}\text { Emulsifier agent needed, adding an extra synthesis } \\
\text { step }\end{array}$ & {$[50-52]$} \\
\hline Core-shell & $\begin{array}{l}\text { Dual property material with outer imprinted } \\
\text { layer (silica, metallic or magnetic) }\end{array}$ & Inefficient imprinting if outer layer is too thin & {$[53-55]$} \\
\hline
\end{tabular}




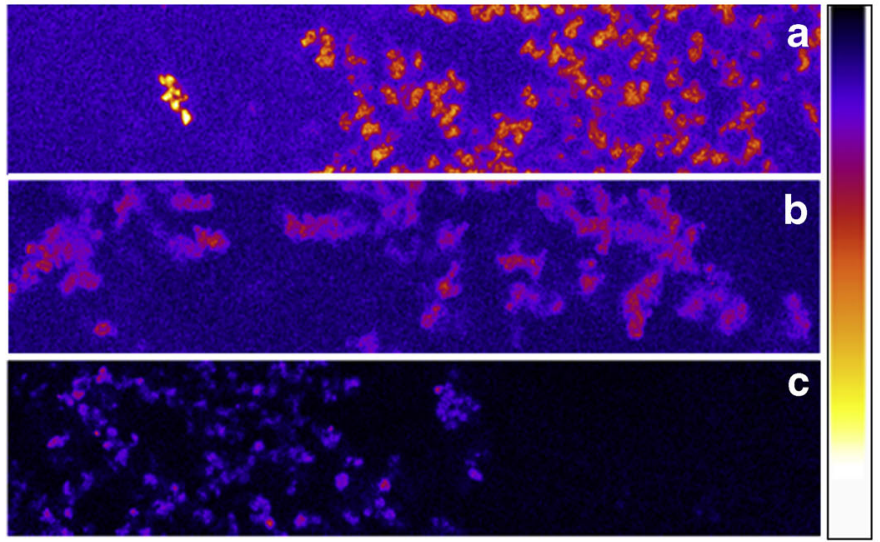

Fig. 3 Confocal microscopy assay to confirm the imprinting effect on the MIP in respect to the NIP. Images of (a) MIP and (b) NIP particles after incubation with histamine and reaction with $o$-phthaldialdehyde (OPA), which yields a maximum fluorescence at $455 \mathrm{~nm}$ upon excitation at

work was published for the detection of BPA by incorporating polymerized MIPs into screen-printed carbon electrodes [49].

\section{Emulsion polymerization}

The emulsion approach consists on mixing the organic monomer(s), the template and the cross-linker solution with an aqueous surfactant media, obtaining a micro- or mini-emulsion. In order to supress the barrier between the two media, the emulsion has to be strongly stirred or even sonicated. The main drawback of this approach is the heterogeneity of the imprinting due to the use of water and the surfactant, which can affect the stability of the template-monomer(s) interactions, and the use of chemical surfactants that will require purifying steps. Consequently, this is not a very attractive technique for MIP synthesis. Recent examples in the literature using emulsion approach include the preparation of MIPs for their usage in solid-phase extraction (SPE) of bisphenols from urine samples [50] or florfenicol from milk [51], or the imprinting of testosterone [52].

\section{Core-shell polymerization}

Core-shell polymerization, as the name suggests, implies the polymerization of the shell of the MIP around a core. This core is not necessarily a non-functional core; it may comprise different materials depending of the desired application, e.g. magnetic and metallic nanoparticles or support like glass beads to offer a control imprinted with grafting or chemical grafting. The main advantage of core-shell MIP particles in respect to other approaches is that those offer a better and tunable control of the thickness of the polymeric film, which in turn allows a better access of the analytes to the cavities and a high binding capability of the material. The main drawback

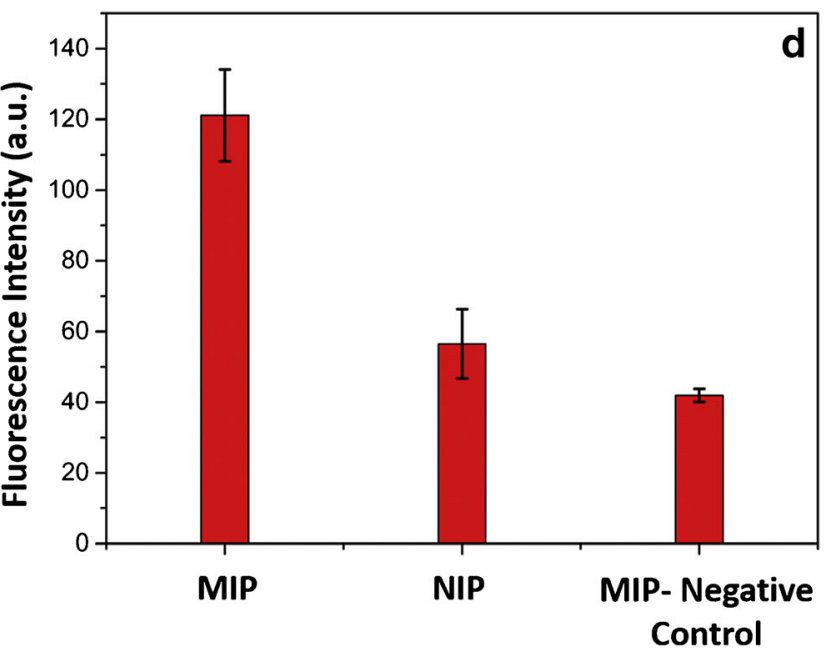

$340 \mathrm{~nm}$. (c) Background fluorescence of the MIP particles when not incubated with histamine, but reacted with OPA, as negative control. (d) Fluorescence intensity of the previous in arbitrary units. Reproduced with permission from Elsevier [48]

is the extra step required for its synthesis and the extra-cost in the laboratory due to the apparatus and the conditions required. The use of magnetic particles as core-shell for a later imprinting is a widely used approach as mentioned by YañezSedeño et al. [53], as it offers the possibility to further preconcentrate the sample and minimize the matrix effects. For example, a magnetic-MIP was prepared for the isolation and detection of biotin and biotinylated biomolecules by ELISA immunoassays [54]. Another interesting approach was the use of $\mathrm{Ru}(\mathrm{phen})_{3}{ }^{2+}$ dye molecules embedded off-centre in the silica core and a thin surface-grafted MIP shell intervening as selective enrofloxacin-binding element for photochemical detection of enrofloxacin [55].

\section{Immobilization}

As abovementioned, the imprinting technique allows obtaining artificial receptors that mimic the biological ones such as enzymes or antibodies. In order to be used for sensing purposes, these materials have to be integrated into the sensor, where a transducer will convert the recognition (or binding) event into an analytical signal. For that purpose, there are two main strategies in the incorporation of MIPs: decoupling the synthesis and immobilization of the artificial receptor (ex situ synthesis) or achieving the direct polymerization onto the electrode surface (in situ). The former enables a better optimization of each step separately, taking often advantage of the immobilization step to incorporate new micro- or nanomaterials to improve the electrochemical response (i.e. to improve the sensitivity or the selectivity). Oppositely, the latter are generally one-step methods, which permit a better 
adherence of the film to the transducer surface and a better control of the film thickness.

In this section, several immobilization strategies used when developing electrochemical sensors will be reviewed. On the one hand, the incorporation of micro- or nano-materials can be achieved by different methods such as drop-, spin- or spraycoating, or through the preparation of composite materials. On the other hand, for the in situ generation of MIP sensors, chemical grafting followed by a polymerization step or electropolymerization are the more common approaches.

\section{Ex situ MIP sensors}

As already stated, decoupling the synthesis and the immobilization steps allows to firstly complete the synthesis and characterization of the MIP particles, and upon selection of the optimum conditions, proceed with their integration into the transducer, being also possible to incorporate at the same time electro-catalytic materials that enable to improve the electrochemical response. Common approaches are mainly based on physical entrapment onto the electrode surface with the aid of some membrane or polymeric material such as sol-gel, Nafion ${ }^{\circledR}$ or polystyrene, or into the composite material that will act as the working electrode by mixing both before its preparation.

\section{Entrapment onto electrode surface}

The incorporation of MIP particles onto the electrode through its entrapment is probably the common approach, as is reflected in several publications. There are several ways by which these could be done, some of the more popular being the use of sol-gel, Nafion ${ }^{\circledR}$, chitosan, agarose, glutaraldehyde, between others. The main advantage of this approach is that it allows the simultaneous incorporation of different materials in a simple and fast way, e.g. MIP beads enhanced with graphene oxide sheets and/or other electrocatalysts. The idea is that the particles are retained into polymeric frameworks, while maintaining their structure and properties. In order to ensure a homogeneous distribution along the sensing platform, several techniques can be used ranging from drop-casting to spin coating (Fig. 4.).

Sol-gel is a method that allows the incorporation of different materials into a generated inorganic framework. The process begins with a colloid liquid suspension of an alkoxide precursor ("sol"), which is gradually transformed into a "gel" by its hydrolysis, poly-condensation and drying [56]. The most common alkoxide precursor is tetraethyl orthosilicate (TEOS) due to the possibility to obtain thin-film porous layers. For example, this approach has been used by Bakas et al. for the entrapment of a bulk MIP via drop-casting when developing an impedimetric sensor for the detection of methidathion [57]. Similarly, several works have been published in our laboratory for the incorporation of different MIP particles into voltammetric sensors via sol-gel immobilization and spin coating $[48,58,59]$.

Nafion ${ }^{\circledR}$ has been widely used for the preparation of modified electrodes due to its convenient properties such as being non-electroactive, hydrophilic and insoluble in water. For the coating of the electrodes, a solution is prepared in alcohol or water, to which nanomaterials can be suspended, deposited onto the electrode surface, by e.g. drop-casting, and finally dried [60]. Following this approach, a hemin-based co-polymerized MIP prepared by precipitation was deposited onto a glassy carbon electrode (GCE) using Nafion ${ }^{\circledR}$ for the amperometric sensing of 4-aminophenol [61]. Similarly, a voltammetric sensor towards tryptophan was prepared by drop-casting of a mixture of the prepared nanoMIP and multi-walled carbon nanotubes (MWCNTs) [62].

Like Nafion ${ }^{\circledR}$, chitosan can be used as membrane to incorporate the MIP nano- or micro-particles as well as different materials. This material is a linear polysaccharide, obtained by deacetylation of chitin that is also commonly used for the preparation of modified sensors due to its biocompatibility, being a toxic-free agent and of lowcost. In this direction, Roushani et al. reported the development of a voltammetric sensor towards manganese based on the casting onto a GCE of the synthetized MIP along with an ionic liquid and MWCNTs via drop-casting from a chitosan mixture [63].

As already stated, there are a large number of polymeric materials suitable to carry out the entrapment of the synthetized MIP particles. For instance, poly(methyl methacrylate) (PMMA) is a synthetic acrylic polymer that can also be used as an entrapment agent. For example, a QCM-based sensor for volatile organic compounds was prepared by spin coating of a dispersion containing the MIP particles and PMMA on an AT quartz crystal resonator [64].

Lastly, electropolymerization of different materials such as poly(3,4-ethylenedioxythiophene) (PEDOT), oPD, pyrrole or Nafion ${ }^{\circledR}$, between others, has also been used not only for the development of electropolymerized MIPs as discussed in the "In situ electropolymerized MIP sensors" section, but also to achieve the immobilization of the MIP particles previously synthetized. The aim is to combine the advantages of decoupling MIP synthesis and immobilisation steps, with the ones from electrochemical preparation [65]. This approach was first reported by Ho et al., who used PEDOT to immobilize the MIP prepared by precipitation polymerization onto an indium tin oxide (ITO) electrode when developing an amperometric sensor towards morphine [66]. Analogous approach has been later adapted with electrochemically grown films of polypyrrole, either after the drop-casting of the MIP particles [65] or directly from a dispersion containing both the particles, single-wall carbon nanotubes (SWCNTs) and pyrrole [67]. 


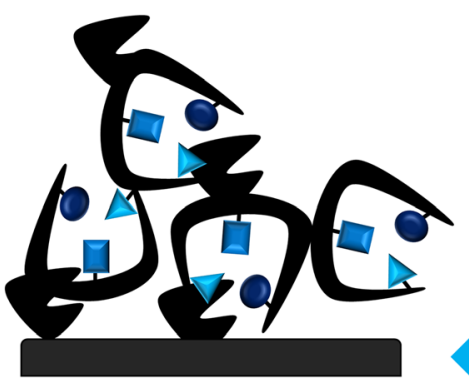

DROP-CASTING

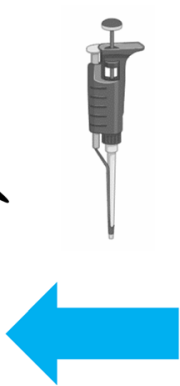

\section{ELECTRODE SURFACE}

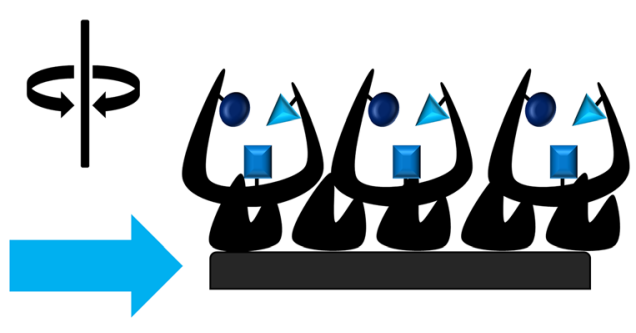

SPIN COATING

Fig. 4 Representation of the immobilization of the MIP particles either by drop-casting or spin coating

\section{Entrapment into the electrode composite}

Another option is the incorporation of the MIP particles directly into the working electrode by mixing the MIP particles with the material used to prepare the electrode. This is possible with, e.g. carbon paste electrodes (CPEs) or graphite-epoxy composites (GECs). The advantage of such an approach is its simplicity and the strong retention of the particles, but unlike the previous polymeric entrapment options, those might offer a smaller number of MIP particles available due to the difference in porosity between the materials. Nevertheless, many examples of such an approach can be found in the literature. For instance, a voltammetric sensor for metronidazole was prepared by mixing the graphite powder, n-eicosane and the MIP particles [68]. Similarly, an impedimetric sensor for the determination of digoxin [69] and a potentiometric sensor for the determination of bisoprolol fumarate [70] have also been reported. Not only the incorporation of MIP particles is possible with traditional sensors, but the deposition of MIPs into electrodes might also be possible by inkjet printing, screen printing, soft lithography, contact printing, 3D printing and roll-to-roll processing [71].

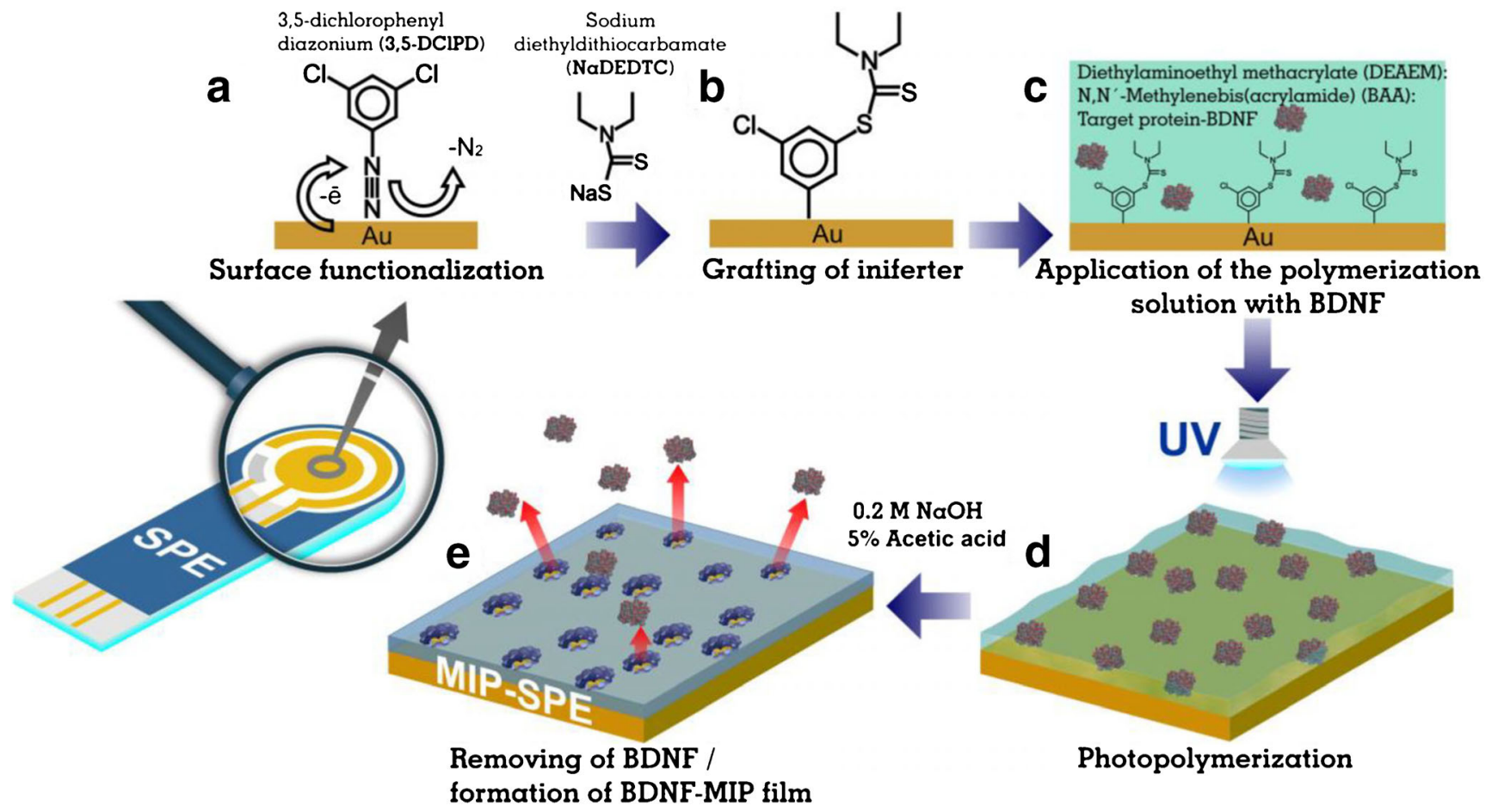

Fig. 5 Schematic of the steps involved in the surface-initiated polymerization. Briefly, the iniferter (which acts as the initiator, transfer agent and terminator) is electrografted to the sensor surface, which is followed by the addition of the solution containing the functional monomer(s), cross- linking agents and template, and the photopolymerization of the monomers under UV irradiation. Reproduced with permission from Estonian Academy Publishers [74] 


\section{In situ MIP sensors}

In order to address the integration and compatibility between the MIP and the transducer, in situ polymerisation of imprinted thin films was proposed. The aim is to promote the formation of selective binding sites closer to the sensor surface and/or enhancing the diffusion kinetics [32]. Furthermore, in situ synthesis provides better adherence of the film to the transducer surface and better control of the film thickness. Over the different approaches that have been proposed, herein we want to highlight two: grafting and electropolymerization.

\section{Grafting}

The attachment of initiators or polymerizable groups through different chemistries has been proposed as a successful approach for the preparation of ultra-thin MIPs [72]. The main methods by which the grafting may be achieved are by utilizing self-assembled monolayers (SAMs) of, e.g. thiols on gold electrodes, silanes or diazonium salts. Next, the polymerization reaction is done directly on the electrode surface, by dropping a solution containing the template, monomer(s), cross-linker(s) or initiator depending on which was the immobilized agent. For example, Khlifi et al. reported the electrografting of 4benzoylphenyl groups from the corresponding diazonium salt, which were used as the photoinitiator for the synthesis of a MIP for melamine detection, offering a covalent binding of the MIP [73]. With a similar approach, Kidakova et al. reported for the first time, a MIP-based synthetic receptor capable of selectively binding a clinically relevant protein - the brain-derived neurotrophic factor (BDNF, Fig. 5) [74].

\section{Electropolymerization}

As already discussed in the "In situ electropolymerized MIP sensors" section, electropolymerization can be used for the in situ synthesis of MIPs. The advantage of such an approach is the better adherence of the film to the transducer surface and control of the film thickness. Earlier, an example using oPD as monomer has already been presented [31]. Similarly, Özcan developed a voltammetric sensor for paracetamol based on a polypyrrole MIP film electrochemically synthetized by simply mixing pyrrole, the template and a supporting electrolyte [75]. With this approach, even MIPs towards bacteria have been synthetized (e.g. S. aureus, Fig. 6) [76]. Another interesting approach is the one proposed by Ma et al. presenting a covalent bond between the protein (template) and glutaraldehyde by imine reaction for determining a prostate-specific antigen (PSA) using a GCE coated with graphene nanoplatelets and gold nanoparticles, further modified with a MIP (Fig. 7) [77].

\section{Applications}

The number of applications involving the usage of MIPs as recognition element for the development of electrochemical sensors is increasingly growing as these polymeric materials gain popularity due to the already discussed advantages derived from their usage. Over the wide range of techniques that electroanalytical methods include, electrochemical sensors are developed based mainly on three types of transduction: potentiometry (where a difference in potential is measured), voltammetry (where a current is measured upon application of a polarization potential) and electrochemical impedance
Fig. 6 Schematic of the sensing approach for $S$. aureus sensing based on an electropolymerized MIP for 3-thiopheneacetic acid and its determination by EIS measurements of the blockage upon rebinding with $\left[\mathrm{Fe}(\mathrm{CN})_{6}\right]^{3 / 4}$ as the redox probe. Reproduced with permission from Elsevier [76]

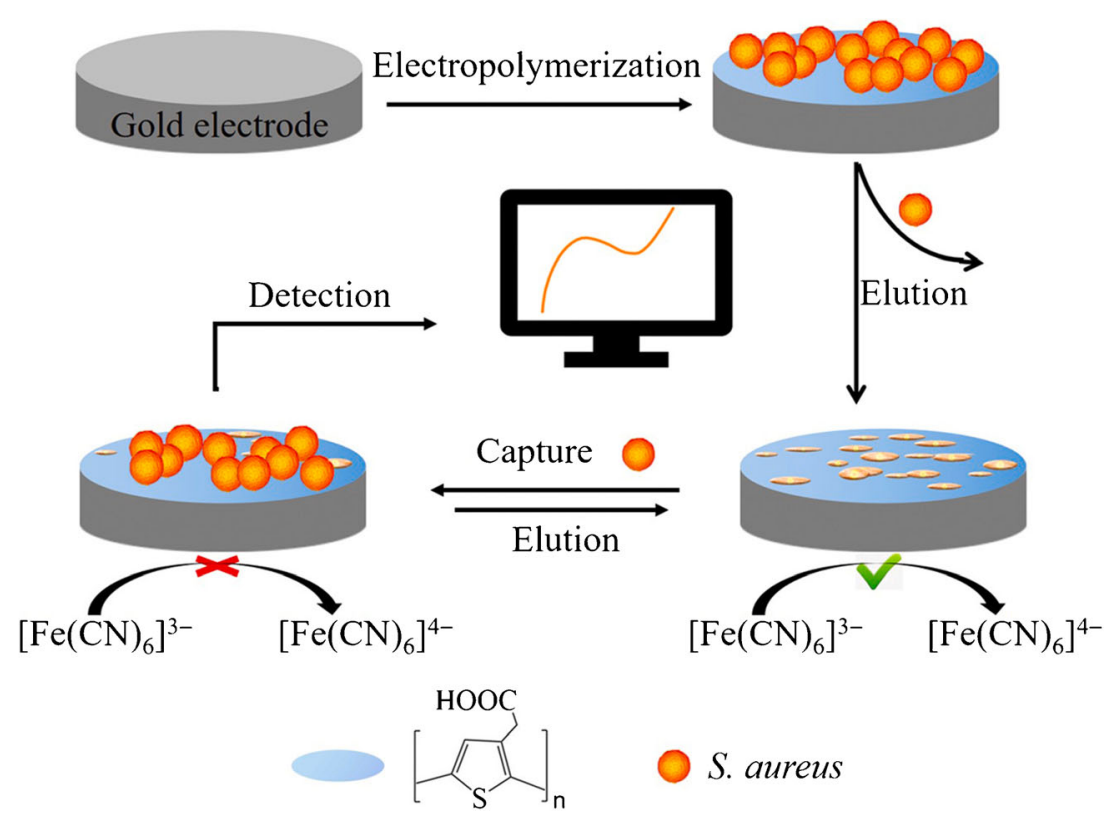


Fig. 7 Schematic of the fabrication and detection principle of the voltammetric MIP-based sensor towards PSA. Briefly, the GCE was modified with a graphene nanosheet-gold nanoparticles (GS-Au) solution, followed by the deposition of chitosan (CS). Next, it was activated with glutaraldehyde (GA) to achieve the covalent immobilization of PSA through an imine bond between the two. Lastly, dopamine was electropolymerized to obtain the in situ MIP after removal of PSA by immersion in an acetic acid solution. Reproduced with permission from Springer [77]

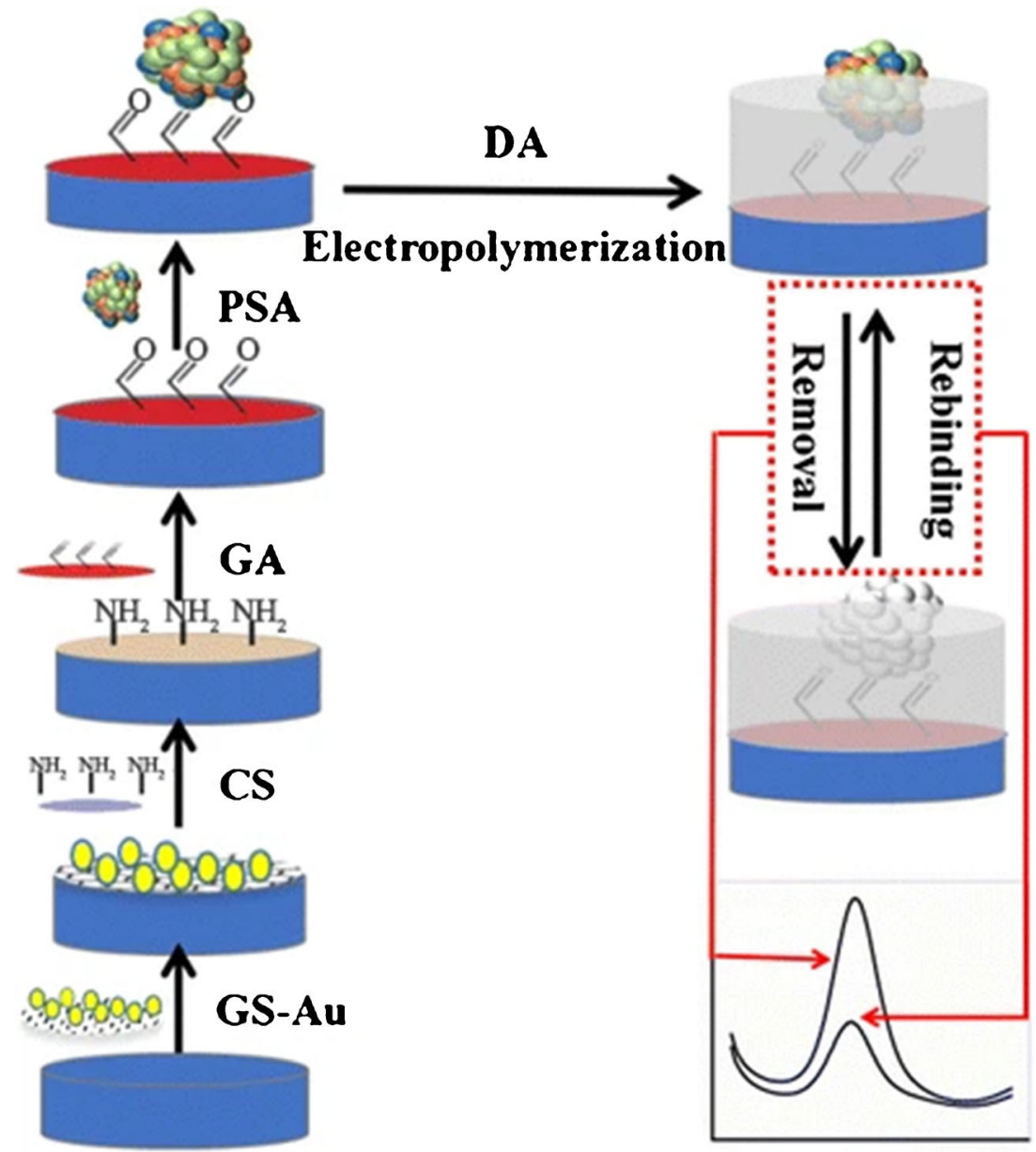

spectroscopy (EIS, where the impedance is measured upon application of different frequency AC potentials). Although each of the techniques presents their inherent advantages and disadvantages $[78,79]$, ultimately the suitability of the chosen technique will depend on the nature of the target analyte and how suitable it is for that technique (e.g. if the compound is electroactive or not) and the sensing strategy (direct detection of the analyte or indirect measurements by measuring the cavities blockage by means of a redox probe such as $\left[\mathrm{Fe}(\mathrm{CN})_{6}\right]^{3 / 4-}$ ).

In this direction, herein we want to present some relevant applications recently reported in the literature over different scenarios in order to illustrate the potential of MIPs in combination with electrochemical sensors (Table 2). However, we want to emphasize that although variants using different techniques and protocols have been included, the aim is not to exhaustively review all the applications, but to demonstrate its wide applicability over different scenarios.

\section{Environmental}

As already introduced, the number of emerging contaminants is growing continuously, and MIPs represent a very interesting approach to allow their on-field detection due to the advantages derived from the combination of MIPs with electrochemical sensors. In this direction, Florea et al. reported the successful development and application of an electrochemical sensor based on an electropolymerized MIP towards the determination of $17 \beta$-estradiol in river waters [80]. Since the target compound is not electroactive, the binding with the MIP was monitored making use of $\left[\mathrm{Fe}(\mathrm{CN})_{6}\right]^{3 / 4-}$ as the redox probe.

Analogously, a MIP-based sensor towards cefalexin (an antibiotic) was also developed, and its application to river water and pharmaceutical samples successfully demonstrated [81]. Interestingly, in this case, the authors compared the performance of the same MIP when using a GCE or a borondoped diamond electrode (BDDE), showing much better performance with the latter, which demonstrates not only the importance of the MIP, but also of the transducer and of the usage of electrocatalysts when developing electrochemical (bio)sensors. Selectivity of the MIP was evaluated over 11 different cephalosporins, showing only significant interference from three of them due to the structural similarities.

In the same direction, Rebelo et al. used quantum mechanical calculations and molecular dynamics simulations to optimize the synthesis of a MIP for furazolidone. Polymers were 


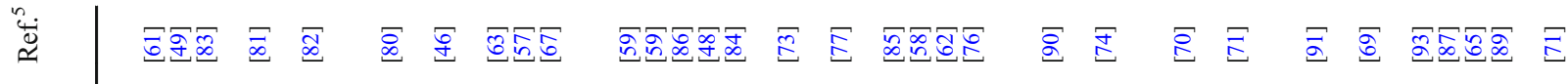

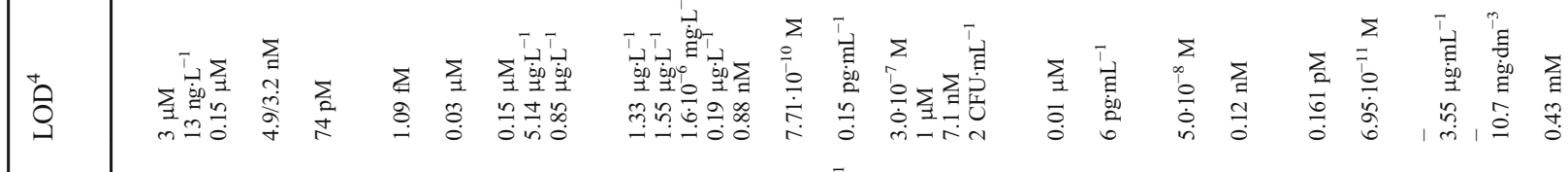

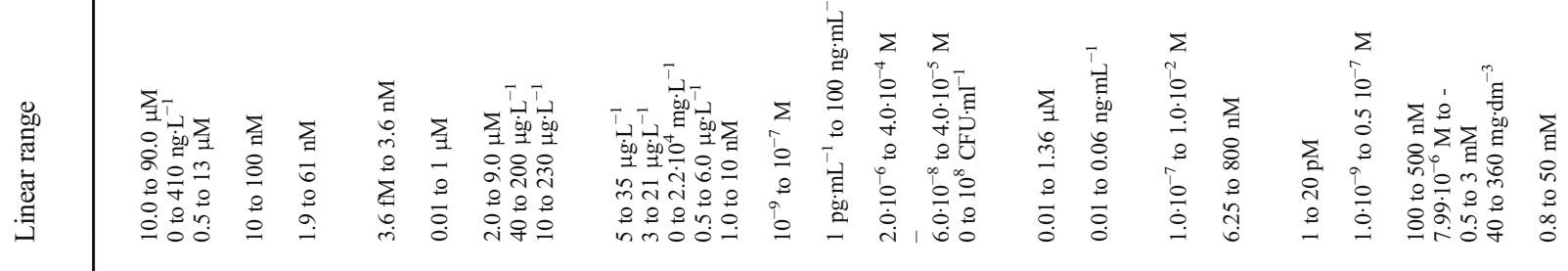

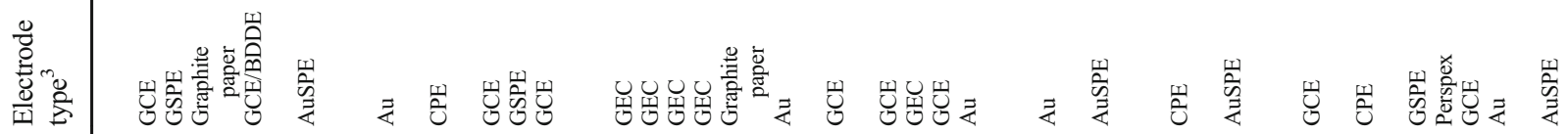
ब.

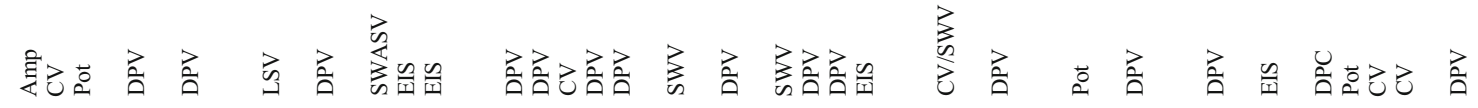

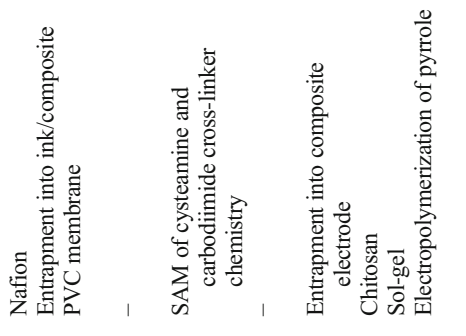

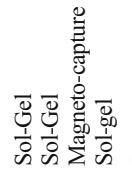
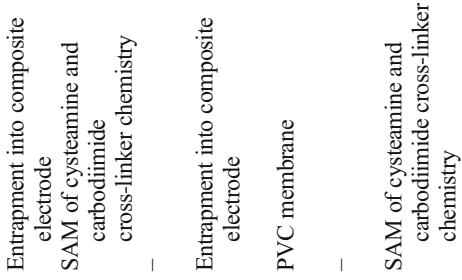

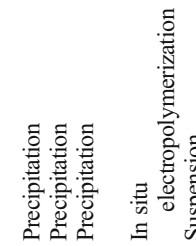
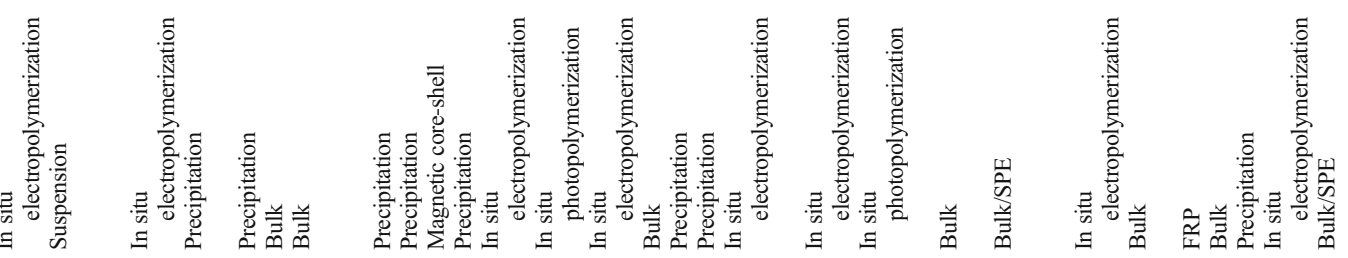

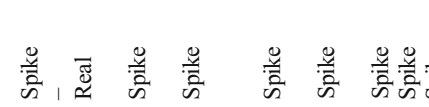

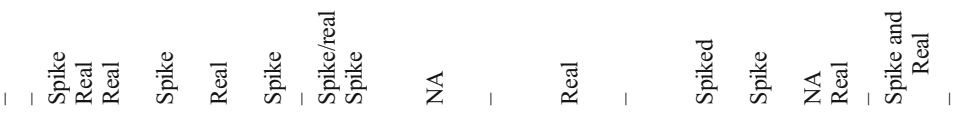

-

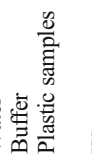
(2) (n) .

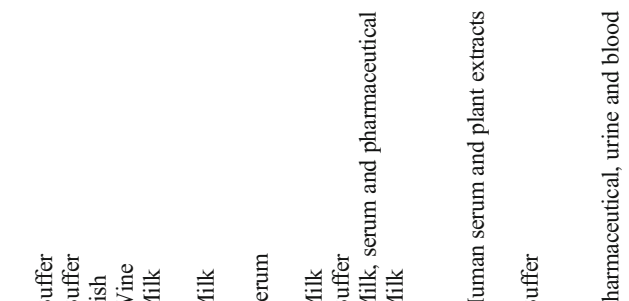

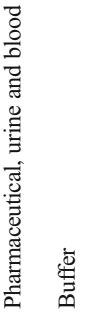
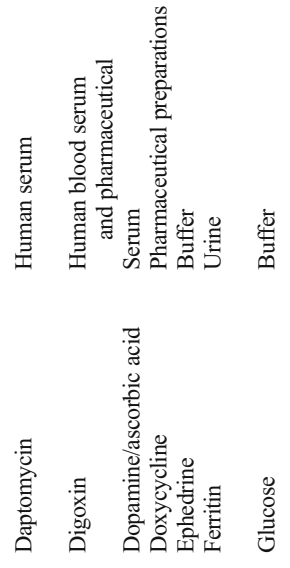


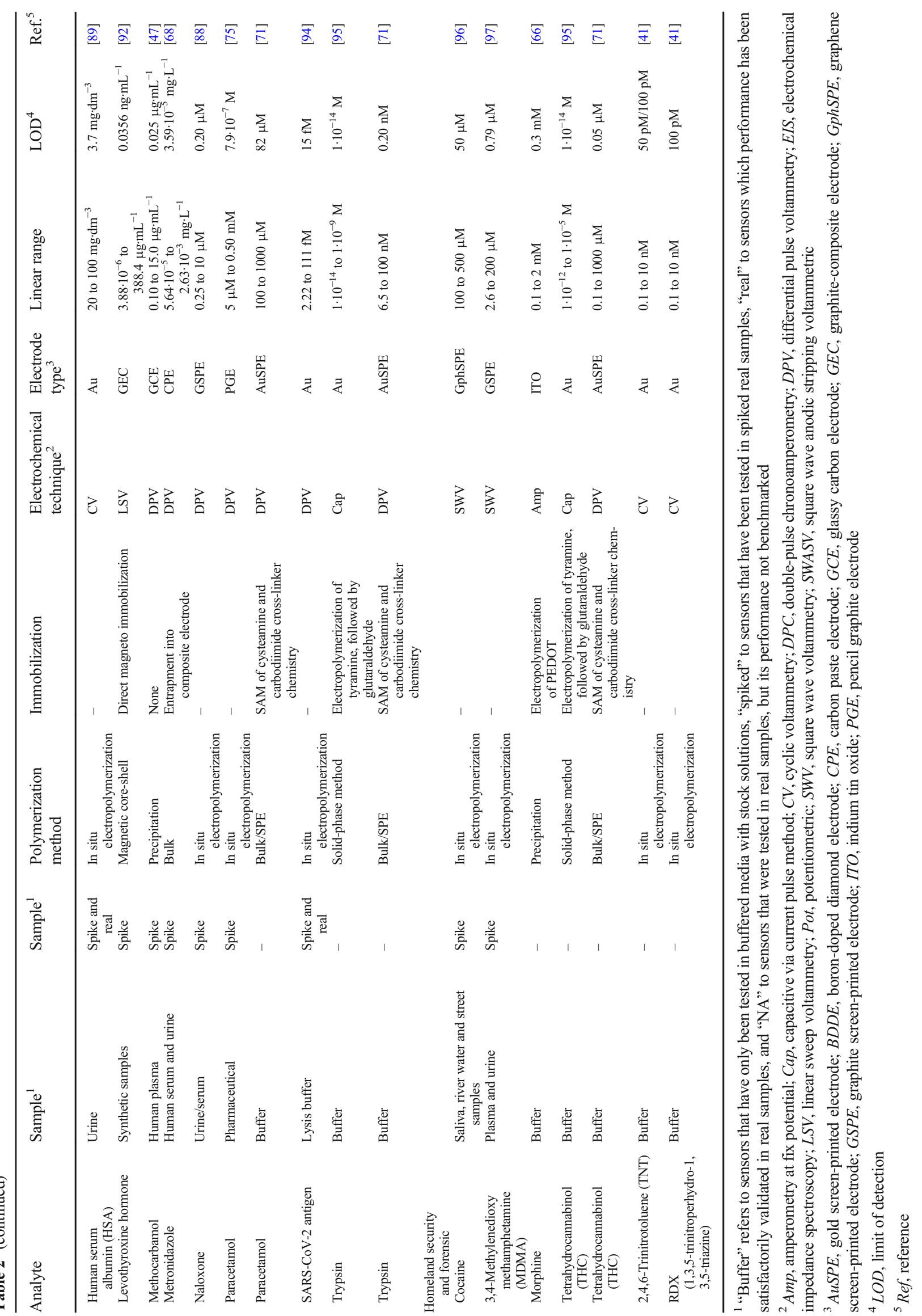


mixed with graphite and MWCNTs to obtain the CPE, which was used to measure the content of furazolidone in tap and river water samples without spiking or doing any pretreatment [46].

In a similar approach, a MIP-based impedimetric sensor was developed for the detection of $N$-nitrosodimethylamine (NDMA) in tap water [67], choosing impedimetric measurements over voltammetric given its higher sensitivity. The MIP particles were first synthetized by the precipitation polymerization method, and next entrapped in an electropolymerized polypyrrole layer. The imprinting effect was confirmed from the sensitivity of the MIP which was 8.3-fold higher than that of the NIP, as well as from the much smaller response (variation of charge transfer resistance) obtained towards structural analogous compounds.

As already stated, MIPs can be even developed towards small compounds as metallic ions. For example, Di Masi et al. reported the synthesis and characterisation of ionimprinted polymer nanoparticles with embedded ligand sites and their application for the electrochemical determination of $\mathrm{Cu}$ (II) [82]. Synthetized MIPs were immobilized on gold screen-printed electrodes (AuSPE) by carbodiimide chemistry after formation of a SAM with cysteamine. The developed MIP showed good response towards $\mathrm{Cu}$ (II), with a sensitivity ca. 7.5 times higher than the NIP (confirming the imprinting), but selectivity coefficients of $0.66-0.68$ for $\mathrm{Ni}$ (II) and $\mathrm{Zn}$ (II), which was attributed to the three of them having a similar ionic radius. In this direction, it has to be highlighted that despite MIPs can be developed towards almost any compound, it is true that the imprinting of smaller compounds is hindered by the more limited interaction sites that those offer.

MIPs have also been used along with potentiometric sensors by mixing the synthetized particles into the ion-selective electrode (ISE) membranes. For example, Kamel et al. reported for the first time a simple and ultra-low-cost, disposable paper-based potentiometric sensor for determination of neutral BPA [83]. Paper substrates are accessible, flexible, recyclable and biodegradable, making them an attractive material for the preparation of electrochemical MIP sensors [83, 84]. The sensors were built employing commercial chromatography paper, into which the synthetized BPA-MIP nanobeads were mixed with the PVC membrane cocktail and incorporated by dropcasting (Fig. 8). Finally, the proposed sensing platform was successfully applied to determine BPA released from real plastic samples.

\section{Food control}

Now in the field of food control, an alternative application was described by Guzmán-Vázquez de Prada et al., in which MIPs were employed as selective solid-phase extraction sorbent and coupled with separate voltammetric detection for the determination of sulfamethazine in milk [85]. Achieving in this manner a significant pre-concentration of the analyte (authors reported an enrichment factor of 45), while also removing possible interferents. In their study, recoveries of ca. 100\% were achieved for the different spiked milk samples.

Another approach to deal with complex samples is the combination of MIPs with magnetic particles, which allow faster pre-concentration while also minimizing matrix effects. For example, Hasan et al. presented the development of a core-shell magnetic-MIP for the electrochemical sensing of histamine in fish samples (Fig. 9) [86]. The developed MIP demonstrated high selectivity as almost no interference was observed for the other three more common biogenic amines. Moreover, recovery values when the sensor was applied to the analysis of fish samples ranged from 96.8 to $102.0 \%$, confirming that this approach can be used for routine food examination.

Recently, da Silva et al. reported the electropolymerization of a MIP on a disposable graphite paper electrode [84]. Concretely, towards the determination of lactose in milk samples (Fig. 10). The MIP showed good imprinting in comparison to the NIP, good selectivity towards structural analogues as well as good repeatability and reproducibility. Even lactose is non-electroactive; detection was accomplished through blocking of conducting channels across the MIP layer and oxidation of a ferrocyanide marker observed by DPV. However, poorer stability was observed for measurements across several days, which is not as bad if considering the disposable nature of the paper sensor.

\section{Clinical diagnostics}

In another example involving potentiometric sensors, Kamel et al. reported the successful determination of doxycycline in pharmaceutical tablets using a MIP-based potentiometric sensor integrated into a flow injection analysis (FIA) system [87]. The sensor was chosen between three different MIPs prepared with different monomers, all of them showing good response, whereas the respective NIP sensors' did not exhibit a linear response in the considered range.

An interesting study presented the development of a MIPbased sensor to be used as a point-of-care device for people with opioid overdose from urine or serum analysis [88]. To this aim, a MIP against naloxone was generated by in situ electropolymerization of 4-aminobenzoic acid (4-ABA) on top of a commercial graphite screen-printed electrode (GSPE) previously modified with MWCNTs. Despite some interference was observed for structural analogous compounds, satisfactory recoveries were obtained for spiked urine and serum samples. Other examples of MIP-based sensors to be used as point-of-care devices have been reported by Stojanovic et al. for the determination of human serum albumin (HSA) and ferritin in urine samples of patients with albuminuria [89], by Waffo et al. for the determination of 


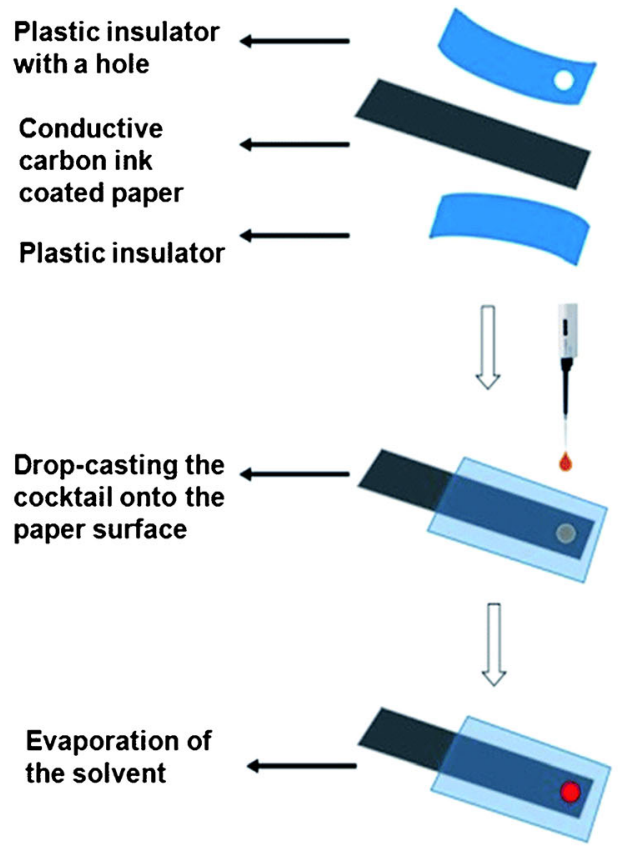

Fig. 8 Schematic representation of the main steps involved in the development of a MIP-based potentiometric sensor for the determination of bisphenol A (BPA). Briefly, the process starts coating chromatography paper with a carbon ink, followed by its coating with an adhesive plastic.

artemisinin in human serum samples [90], or by Ozcelikay et al. for the determination of daptomycin also in human serum samples [91].

A complete study for the selective recognition of levothyroxine hormone with a magneto-actuated immunoassay and electrochemical sensing was presented by Moura et al. [92]. Firstly, theoretical studies were carried out by DFT to choose the best monomers, and among those, the performance of the best three candidates was experimentally compared. Finally, the selected MIP was used to develop the electrochemical sensor which was compared with the gold standard
Finally, the membrane cocktail containing the MIP particles was dropcasted onto the build sensor surface. Reproduced with permission from Royal Society of Chemistry [83]

method providing a thoughtful strategy for replacing antibodies.

A particular application was described by Rapini et al. where a nanoMIP was used as sequestering (masking) agent, to suppress the signal coming from interfering molecules and facilitate the detection of the target analyte [93]. Concretely, suppression of ascorbic acid interference, when attempting the determination of dopamine in serum samples, was achieved by adding the nanoMIP into the electrochemical cell when the concentration of the former was under $50 \mathrm{nM}$. Besides the actual results, what is especially significant is the novelty of

a

b

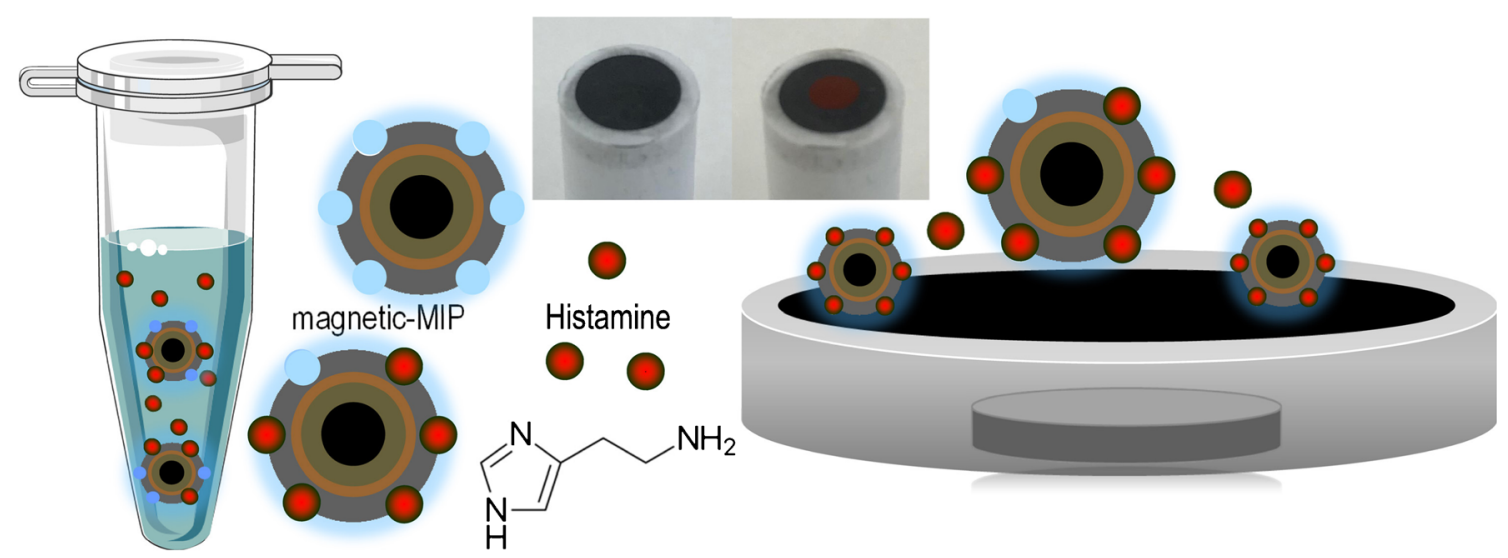

Fig. 9 Schematic illustration of the pre-concentration and electrochemical sensing of histamine based on the usage of a magnetic-MIP and a magneticGEC electrode. Reproduced with permission from Elsevier [86] 

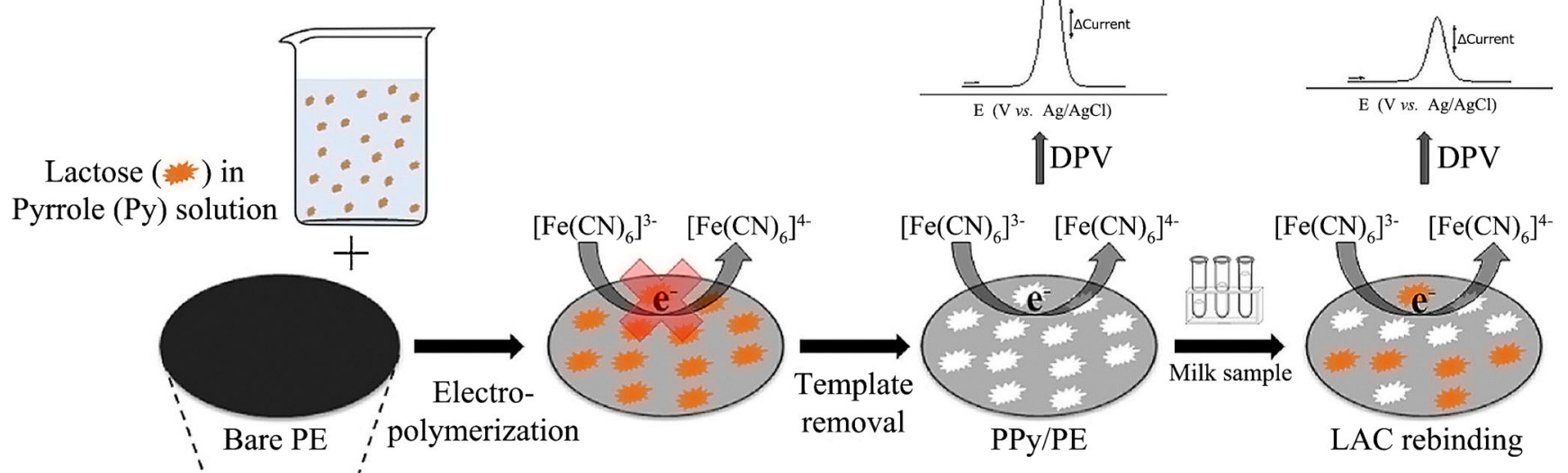

PPy/PE - Polypyrrole (PPy) molecularly imprinted on graphite paper electrode (PE).

Fig. 10 Schematic representation of the main steps involved in the development of a MIP-based voltammetric sensor for the determination of lactose. Briefly, the process starts with the electropolymerization of pyrrole in the presence of lactose. Next, the template is removed by overoxidation in a $\mathrm{NaOH}$ solution, leaving three-dimensional imprinted

the application itself, which can be extrapolated to other analytes.

Lastly, in a very hot topic nowadays with the current COVID-19 pandemic caused by SARS-CoV-2 coronavirus, Raziq et al. reported the development of an accurate and cheap portable sensor based on a MIP-based voltammetric sensor and the usage of a portable compact potentiostat which can be controlled through a smartphone (Fig. 11) [94]; all of them crucially important characteristics for the clinical diagnosis. The MIP sensor was able to signal the presence of SARSCoV-2 nucleoprotein (ncovNP) in nasopharyngeal swab samples of COVID-19-positive patients, unlocking in this manner new routes for the development of rapid COVID-19 diagnostic tools.

\section{Homeland security and forensic}

Canfarotta et al. reported a manufacturing-friendly protocol for the synthesis of MIP nanoparticles produced by solidphase synthesis and their integration into label-free capacitive sensors [95]. The latter chosen due to the higher sensitivity that those offer compared with other techniques, as well as offering label-free measurements. To prove the feasibility of the approach, two different target analytes with different molecular weight were evaluated: tetrahydrocannabinol (THC) and trypsin (a protein). Significant performance was obtained for trypsin, with limits of detections (LODs) down to $10^{-14}$ and high selectivity compared to the response obtained for other proteins, while the performance of THC-MIP was not cavities complementary to the template. Sensing is based on the measurement of pore blockage with $\left[\mathrm{FE}(\mathrm{CN})_{6}\right]^{3-}$ upon rebinding of lactose when the sensor is incubated with the samples. Reproduced with permission from Elsevier [84]

as good as that for the protein, as could be expected due to the difference in size between both molecules and, consequently, the number of functional groups to generate the imprint.

Many other examples can be found for the detection of illicit drugs. A remarkable study was published by Florea et al. [96] for the sensing of cocaine in real samples. The MIP was based on the in situ electropolymerization of $p$ aminobenzoic acid (which was selected by computational modelling) onto graphene-modified electrodes with palladium nanoparticles integrated into the sensing layer to enhance the number of accessible imprinted sites, to improve their homogeneous distribution and to provide a catalytic effect. The selectivity of the developed MIP was demonstrated against homatropine (an alkaloid with similar structure) showing no response, and recoveries of ca. $100 \%$ in spiked river water and saliva.

Similarly, the detection of 3,4 methylenedioxymethamphetamine (MDMA) has been achieved by using $\mathrm{OPD}$ as the monomer to be electropolymerized onto GSPE, chosen also after DFT calculations to optimize the polymer structure as well as the interactions of the monomer and the template [97]. The MIP-based sensor showed good selectivity, showing only dopamine and tyramine some binding with the MIP, but being eletroanalytically distinguishable. This was confirmed from the analysis of spiked urine and plasma samples, where recoveries of $81-91 \%$ were obtained.

The detection of explosive compounds is another hot topic in forensic analysis due to the surge of international terrorism and the increased use of explosives in terrorist attacks. In 


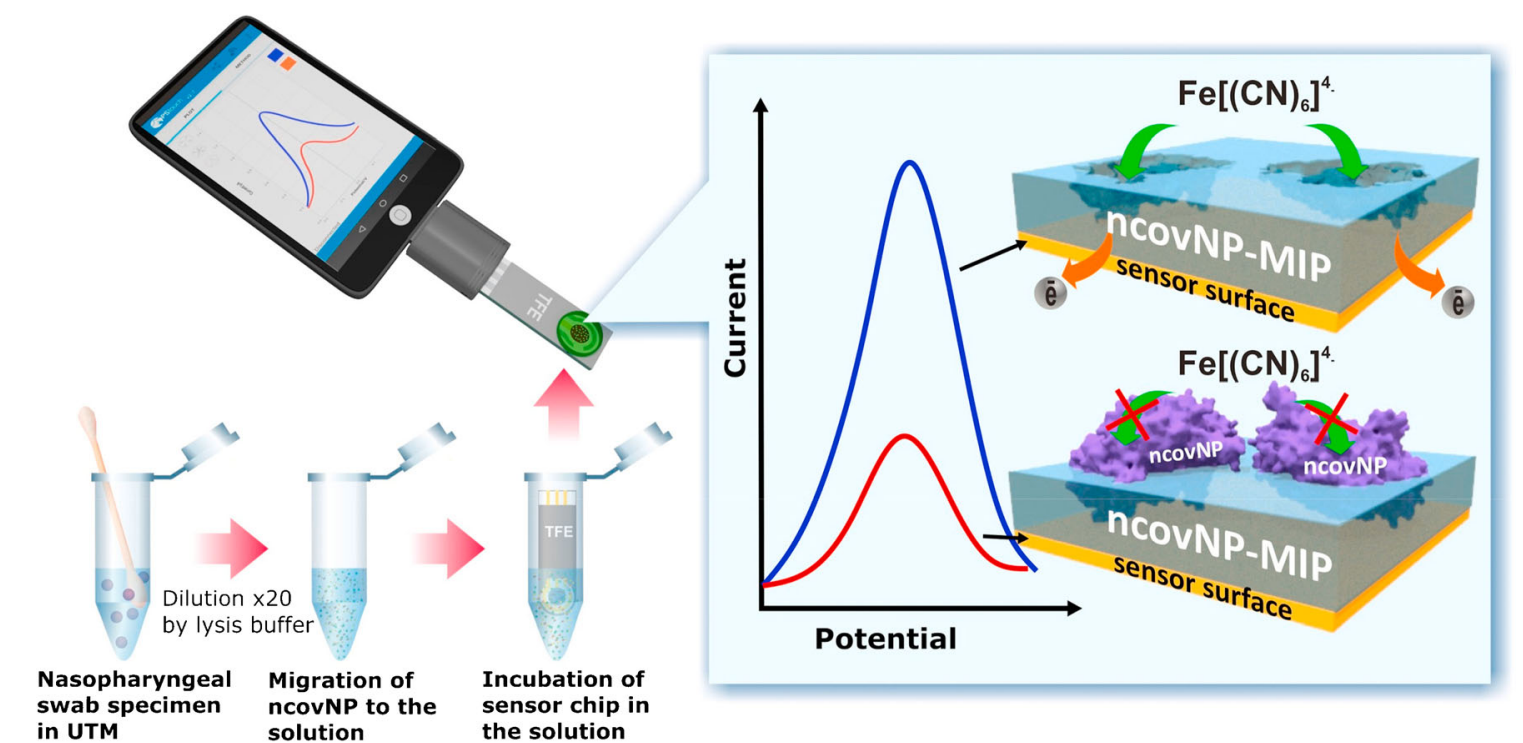

Fig. 11 Schematic of the steps of the COVID-19 diagnostics principle by ncovNP sensor analysing the samples prepared from nasopharyngeal swab specimens of patients, and of the electrochemical measurement

based on pore blockage of the $\left[\mathrm{Fe}(\mathrm{CN})_{6}\right]^{4-}$. Reproduced with permission from Elsevier [94]

addition, the inherent redox activity of nitro-aromatic compounds makes them ideal candidates for electrochemical methods thanks to their multi-peak reduction signal [98]. In this regard, Leibl et al. described the electropolymerization of two dummy MIPs for 2,4,6-trinitrotoluene (TNT) and RDX (1,3,5-trinitroperhydro-1,3,5-triazine) using trimesic acid and Kemp's triacid as structural analogues [41]. Moreover, the choice of dopamine as the functional monomer was based on DFT computational design using the binding energies for complex formation with both the dummy and the actual target as ranking parameter. The developed MIP-based sensor allowed for a $10^{5}$-fold sensitivity improvement over a bare gold electrode, demonstrating the advantage derived from the usage of MIPs.

\section{Conclusions and future trends}

The combination of MIPs with electrochemical sensors for the development of new sensing devices has been explored in this review. More specifically, an overview of the more common synthetic approaches as well as the strategies that can be used to achieve their integration has been presented, followed by some recent examples over different areas in order to illustrate the potential of such combination in very diverse applications. Overall, demonstrating the advantages that can be derived from the usage of MIPs, which show comparable performance to affinity receptors such as enzymes or antibodies in terms of selectivity, but with the added advantage of its higher stability, lower cost, tunability to almost any template and, more importantly, more versatile option in terms of operation conditions (e.g. extreme temperatures or $\mathrm{pH}$ levels and organic solvents).

As a result of the interest that MIPs have arisen, significant progress has been made over the last two decades in the development of new synthetic and polymerization approaches that enhance the performance of the obtained polymers. In this direction, it must be highlighted the emergence of electropolymerization which allows a facile approach for the obtaining of electrochemical-based MIPs, the role of computational approaches which allow a rational design of MIPs, reducing significantly the experimental screening of the best candidate, or the appearance of alternative synthetic approaches such as dummy MIPs, which provide an experimental alternative to obtain MIPs towards specific analytes that otherwise would be cumbersome. Furthermore, the combination of nanomaterials with MIPs and sensors allows enhancing the properties of the synthetized polymers as well as the electrochemical response of the latter, giving rise to hybrid materials with new properties and higher potential. For example, recently, Piletsky et al. reported on a nanoMIP that incorporates ferrocene as the redox probe, which upon binding of the template generates a conformational change that can be detected electrochemically even if the target analyte is nonelectroactive (Fig. 12) [71, 99]. Precisely, the latter is a good example of the efforts made to produce protocols that allow this technology to reach the market, which as discussed in more detail by Lowdon et al., is being hindered by a combination of factors such as the challenge that represents the scale-up production process or the fact that lab-based analysis using techniques such as liquid or gas chromatography remain as the gold standard [100].

However, despite the huge potential that MIPs have demonstrated, it can be neglected that as any sensor or biosensor, those might still show certain cross-response and/or suffer 
Fig. 12 (Top) Schematic representation of the response principle of the MIP-based sensor. Briefly, the rebinding of the analyte to the MIP triggers a detectable change in the polymer conformation thanks to the ferrocene that is incorporated into the polymeric matrix. (Bottom) Scanning electron microscopy images of the synthetized MIPs for paracetamol detection. Reproduced with permission from Springer [71]
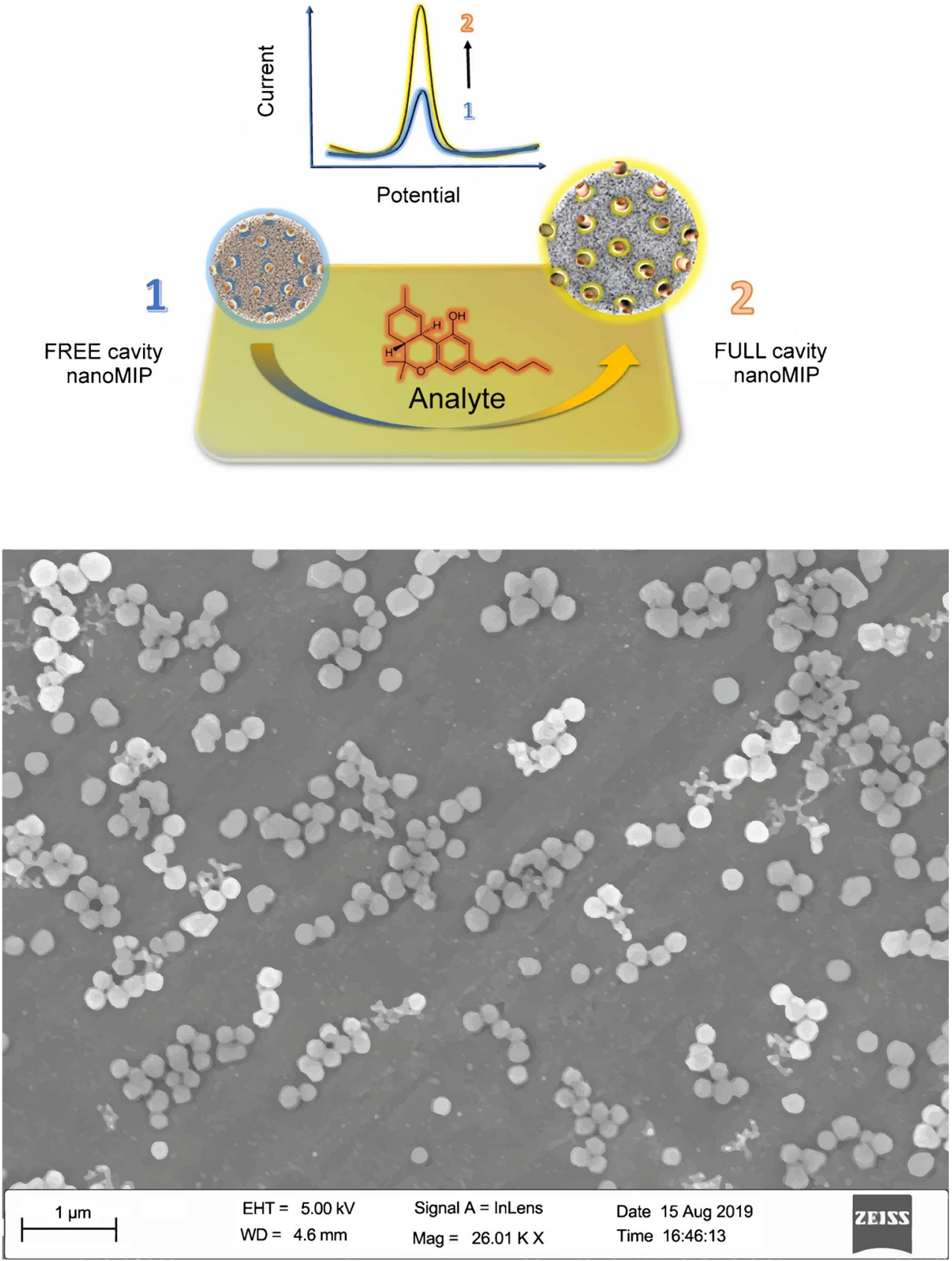

from undesired matrix effects. To overcome such issues, the straightforward thought is on trying to improve the synthetized material, but higher benefits can be even obtained if applying chemometric methods (an approach known as electronic tongue, ET).

Such an approach was first reported in 1985, when Otto and Thomas attempted the determination of free metal ions with an array of non-specific ISEs, and demonstrated the advantages that could be obtained from the use of partial least squares regression (PLS) in comparison to ordinary least squares regression (OLS) [101]. A few years later, the same approach was employed to improve the performance of biosensors, in what has been referred to as bioelectronic tongue (BioET) [102, 103].

An ET is a biomimetic system applied to the analysis of liquid samples, which consists in the coupling of an array of sensors capable of giving a wide and complete response of the analysed species, plus a chemometric processing tool able to interpret and extract meaningful data from the complex readings [102, 104]. BioETs only differ from ETs in the fact that one or several biosensors are combined into the ET sensor array, commonly sharing the same transduction principle to facilitate their compatibility. 


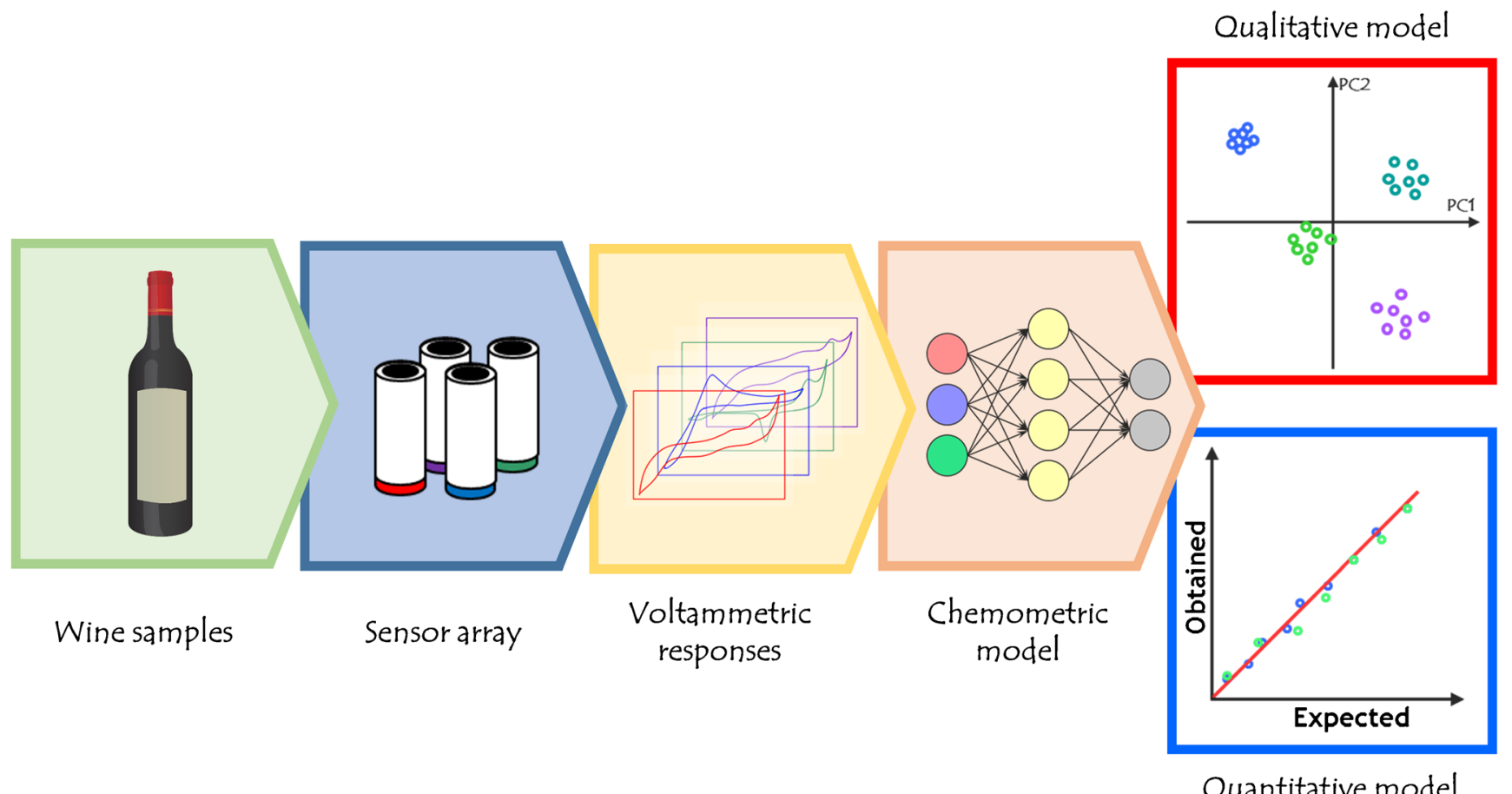

Fig. 13 Schematic representation of the steps involved in the ET approach reported by Herrera-Chacon et al. [59]. Briefly, the set of samples are measured with the MIP-based sensor array, collecting a whole

In this direction, the incorporation of MIP-based sensors into ET sensor arrays would be of great interest, due to the already discussed advantages of the former, allowing to unleash new applications and opening the room for the development of what could be considered a new type of ETs based on its usage. On the one side, the incorporation of MIP-based sensors into ETs allows to address the lack of selectivity of the latter in a similar way that biosensors do, but offering lower cost systems with higher stability and operability in harsher conditions, while allowing to obtain a suitable receptor towards almost any analyte of interest. On the other side, the modelling stage involved in ETs allows to solve possible interferences and to improve the selectivity of the synthetized MIPs, by shifting the complexity from the chemical to the modelling side [102, 104, 105]. However, despite the high number of publications related to the development of MIPbased electrochemical sensors, very few publications can be found exploiting this approach [106].

One of those few studies is the one from Bueno et al. where an array of four hydrogel-based MIPs towards different proteins (bovine haemoglobin, equine myoglobin, cytochrome $\mathrm{C}$ and bovine serum albumin) was prepared using GCEs. The voltammetric responses were submitted to PCA in order to attempt their discrimination, showing clear clusters for each of the proteins. However, despite the same trend was observed for the NIP, it opened the door for future applications [107]. Precisely, in a very similar approach with spiked samples, El- voltammogram for each of the samples. Next, the data is processed by means of PCA to achieve the discrimination of the different volatile phenols or ANNs for the quantification of volatile phenols mixtures

Sharif et al. showed the potential of using haemoglobyn MIPs for differentiating meat species [108]. In another study, Chatterjee et al. developed a polyacrylamide MIP sensor towards theaflavin, and attempted the correlation of the voltammetric readings with the theaflavin content estimated by HPLC by means of partial least squares regression (PLS) employing a leave-one-out (LOO) approach; the study demonstrated good agreement between both, although further validation might be required due to the reduced number of samples and the use of LOO validation [109]. Lastly, in our laboratory, the application of a MIP-based ET towards the determination of volatile phenols in wines with the Brett defect was also reported [59]. In this case, the ET array was formed by 3 sensors modified with a MIP towards 4-ethylphenol, another towards 4-ethylguaiacol and the NIP, achieving the resolution and individual quantification of binary mixtures of both volatile phenols by means of artificial neural networks (ANNs) (Fig. 13).

Funding The authors acknowledge the support of the Spanish Ministry of Science and Innovation (MCINN) through project PID2019107102RB-C21. A.H.C. thanks the support of the projects CTQ-201680170-P. M.d.V. thanks the support from Generalitat de Catalunya through the program ICREA Academia.

Data availability Not applicable.

Code availability Not applicable. 


\section{Declarations}

Conflict of interest The authors declare no competing interests.

\section{References}

1. Agüera A, Martínez Bueno MJ, Fernández-Alba AR. New trends in the analytical determination of emerging contaminants and their transformation products in environmental waters. Environ Sci Pollut Res. 2013;20(6):3496-515.

2. Galindo-Miranda JM, Guízar-González C, Becerril-Bravo EJ, Moeller-Chávez G, León-Becerril E, Vallejo-Rodríguez R. Occurrence of emerging contaminants in environmental surface waters and their analytical methodology - a review. Water Sci Technol Water Supply. 2019;19(7):1871-84.

3. Peltomaa R, Benito-Peña E, Gorris HH, Moreno-Bondi MC. Biosensing based on upconversion nanoparticles for food quality and safety applications. Analyst. 2021;146(1):13-32.

4. Mani V, Beduk T, Khushaim W, Ceylan AE, Timur S, Wolfbeis OS, et al. Electrochemical sensors targeting salivary biomarkers: a comprehensive review. TrAC - Trends Anal Chem. 2021;135: 116164

5. Wang J. Portable electrochemical systems. TrAC - Trends Anal Chem. 2002;21(4):226-32.

6. Luo X, Morrin A, Killard AJ, Smyth MR. Application of nanoparticles in electrochemical sensors and biosensors. Electroanalysis. 2006;18(4):319-26.

7. Walcarius A, Minteer SD, Wang J, Lin Y, Merkoçi A. Nanomaterials for bio-functionalized electrodes: recent trends. J Mater Chem B. 2013;1(38):4878-908.

8. Arduini F, Micheli L, Moscone D, Palleschi G, Piermarini S, Ricci $\mathrm{F}$, et al. Electrochemical biosensors based on nanomodified screen-printed electrodes: recent applications in clinical analysis. TrAC Trends Anal Chem. 2016;79:114-26.

9. Chen A, Chatterjee S. Nanomaterials based electrochemical sensors for biomedical applications. Chem Soc Rev. 2013;42(12): $5425-38$

10. Maduraiveeran G, Sasidharan M, Ganesan V. Electrochemical sensor and biosensor platforms based on advanced nanomaterials for biological and biomedical applications. Biosens Bioelectron. 2018;103:113-29.

11. Zhang X, Li G, Wu D, Zhang B, Hu N, Wang H, et al. Recent advances in the construction of functionalized covalent organic frameworks and their applications to sensing. Biosens Bioelectron. 2019 Dec;145(July):111699.

12. Fischer E. Einfluss der configuration auf die wirkung der enzyme. Ber Dtsch Chem Ges. 1894 Oct;27(3):2985-93.

13. Polyakov MV. Adsorption properties and structure of silica gel. Zhurnal Fizieskoj Khimii/Akad. 1931;2:799-805.

14. Breinl FHF. Chemical examinations on the precipitate from haemoglobin and anti-haemoglobin serum and comments on the nature of antibodies. ZPhysiol Chem. 1930;192:45-57.

15. Pauling L. A theory of the structure and process of formation of antibodies. J Am Chem Soc. 1940;62(10):2643-57.

16. Dickey FH. The preparation of specific adsorbents. Proc Natl Acad Sci U S A. 1949;35:227-9.

17. Martin AJP. In Nobel lectures, chemistry. Elsevier. 19421962;1952:359-71.

18. Takagishi T, Klotz IM. Macromolecule-small molecule interactions; introduction of additional binding sites in polyethyleneimine by disulfide cross-linkages. Biopolymers. 1972;11(2):483-91.
19. Wulff G, Sarhan A. Use of polymers with enzyme-analogous structures for resolution of racemates. Angew Chem Int Ed. 1972;11:341-4.

20. Sagiv J. Organized monolayers by adsorption. III. Irreversible adsorption and memory effects in skeletonized silane monolayers. Isr J Chem. 1979;18(3-4):346-53.

21. Whitcombe MJ, Kirsch N, Nicholls IA. Molecular imprinting science and technology: a survey of the literature for the years 2004 2011. J Mol Recognit. 2014;27:297-401.

22. Alexander C, Andersson HS, Andersson LI, Ansell RJ, Kirsch N, Nicholls IA, et al. Molecular imprinting science and technology: a survey of the literature for the years up to and including 2003. J Mol Recognit. 2006;19(2):106-80.

23. Wulff G, Vesper W, Grobe-Einsler R, Sarhan A. Enzymeanalogue built polymers, 4 . On the synthesis of polymers containing chiral cavities and their use for the resolution of racemates. Makromol Chem Macromol Chem Phys. 1977;178:2799-816.

24. Andersson LI, Mosbach K. Enantiomeric resolution on molecularly imprinted polymers prepared with only non-covalent and non-ionic interactions. J Chromatogr A. 1990;516(2):313-22.

25. Leonhardt A, Mosbach K. Enzyme-mimicking polymers exhibiting specific substrate binding and catalytic functions. React Polym Ion Exch Sorbents. 1987;6(2-3):285-90.

26. Norrlöw O, Glad M, Mosbach K. Acrylic polymer preparations containing recognition sites obtained by imprinting with substrates. J Chromatogr A. 1984;299(C):29-41.

27. Haupt K, Mosbach K. Molecularly imprinted polymers and their use in biomimetic sensors. Chem Rev. 2000;100(7):2495-504.

28. Wulff G, Sarhan A, Zabrocki K. Enzyme-analogue built polymers and their use for the resolution of racemates. Tetrahedron Lett. 1973;14(44):4329-32.

29. Whitcombe MJ, Rodriguez ME, Villar P, Vulfson EN. A new method for the introduction of recognition site functionality into polymers prepared by molecular imprinting: synthesis and characterization of polymeric receptors for cholesterol. J Am Chem Soc. 1995;117(27):7105-11.

30. Fujii Y, Matsutani K, Kikuchi K. Formation of a specific coordination cavity for a chiral amino acid by template synthesis of a polymer Schiff base cobalt(III) complex. J Chem Soc Chem Commun. 1985;7(7):415-7.

31. Malitesta C, Losito I, Zambonin PG. Molecularly imprinted electrosynthesized polymers: new materials for biomimetic sensors. Anal Chem. 1999;71(7):1366-70.

32. Malitesta C, Mazzotta E, Picca RA, Poma A, Chianella I, Piletsky SA. MIP sensors - the electrochemical approach. Anal Bioanal Chem. 2012;402(5):1827-46.

33. Cowen T, Karim K, Piletsky S. Computational approaches in the design of synthetic receptors - a review. Anal Chim Acta. 2016;936:62-74.

34. Karim K, Breton F, Rouillon R, Piletska EV, Guerreiro A, Chianella I, et al. How to find effective functional monomers for effective molecularly imprinted polymers? Adv Drug Deliv Rev. 2005;57(12):1795-808.

35. Nicholls IA, Andersson HS, Charlton C, Henschel H, Karlsson BCG, Karlsson JG, et al. Theoretical and computational strategies for rational molecularly imprinted polymer design. Biosens Bioelectron. 2009;25(3):543-52.

36. Bates F, Busato M, Piletska E, Whitcombe MJ, Karim K, Guerreiro A, et al. Computational design of molecularly imprinted polymer for direct detection of melamine in milk. Sep Sci Technol. 2017;52(8):1441-53.

37. Sergeyeva TA, Piletsky SA, Brovko AA, Slinchenko EA, Sergeeva LM, El'skaya AV. Selective recognition of atrazine by molecularly imprinted polymer membranes. Development of conductometric sensor for herbicides detection. Anal Chim Acta. 1999;392(2-3):105-11. 
38. Xu D, Zhu W, Jiang Y, Li X, Li W, Cui J, et al. Rational design of molecularly imprinted photonic films assisted by chemometrics. J Mater Chem. 2012;22(32):16572-81.

39. Rosengren AM, Golker K, Karlsson JG, Nicholls IA. Dielectric constants are not enough: principal component analysis of the influence of solvent properties on molecularly imprinted polymer-ligand rebinding. Biosens Bioelectron. 2009;25(3):5537.

40. Sellergren B, Andersson L. Molecular recognition in macroporous polymers prepared by a substrate analog imprinting strategy. J Organomet Chem. 1990 May;55(10):3381-3.

41. Leibl N, Duma L, Gonzato C, Haupt K. Polydopamine-based molecularly imprinted thin films for electro-chemical sensing of nitro-explosives in aqueous solutions. Bioelectrochemistry. 2020;135:107541.

42. Yin YM, Chen YP, Wang XF, Liu Y, Liu HL, Xie MX. Dummy molecularly imprinted polymers on silica particles for selective solid-phase extraction of tetrabromobisphenol A from water samples. J Chromatogr A. 2012;1220:7-13.

43. Zahedi P, Ziaee M, Abdouss M, Farazin A, Mizaikoff B. Biomacromolecule template-based molecularly imprinted polymers with an emphasis on their synthesis strategies: a review. Polym Adv Technol. 2016;27(9):1124-42.

44. Urraca JL, Marazuela MD, Merino ER, Orellana G, MorenoBondi MC. Molecularly imprinted polymers with a streamlined mimic for zearalenone analysis. J Chromatogr A. 2006;1116(12):127-34.

45. Benito-Peña E, Martins S, Orellana G, Moreno-Bondi MC. Watercompatible molecularly imprinted polymer for the selective recognition of fluoroquinolone antibiotics in biological samples. Anal Bioanal Chem. 2009;393(1):235-45.

46. Rebelo P, Pacheco JG, Voroshylova I V., Melo A, Cordeiro MNDS, Delerue-Matos C. Rational development of molecular imprinted carbon paste electrode for Furazolidone detection: theoretical and experimental approach. Sensors Actuators B Chem. 2021;329(20).

47. Gholivand MB, Khodadadian M. Rationally designed molecularly imprinted polymers for selective extraction of methocarbamol from human plasma. Talanta. 2011;85(3):1680-8.

48. Herrera-Chacón A, Dinç-Zor Ș, del Valle M. Integrating molecularly imprinted polymer beads in graphite-epoxy electrodes for the voltammetric biosensing of histamine in wines. Talanta. 2020;208:120348.

49. Ekomo VM, Branger C, Bikanga R, Florea AM, Istamboulie G, Calas-Blanchard C, et al. Detection of Bisphenol A in aqueous medium by screen printed carbon electrodes incorporating electrochemical molecularly imprinted polymers. Biosens Bioelectron. 2018;112(April):156-61.

50. Yang J, Li Y, Wang J, Sun X, Cao R, Sun H, et al. Molecularly imprinted polymer microspheres prepared by Pickering emulsion polymerization for selective solid-phase extraction of eight bisphenols from human urine samples. Anal Chim Acta. 2015;872:35-45.

51. Chen H, Son S, Zhang F, Yan J, Li Y, Ding H, et al. Rapid preparation of molecularly imprinted polymers by microwaveassisted emulsion polymerization for the extraction of florfenicol in milk. J Chromatogr B Anal Technol Biomed Life Sci. 2015;983-984:32-8.

52. Kellens E, Bové H, Conradi M, D’Olieslaeger L, Wagner P, Landfester K, et al. Improved molecular imprinting based on colloidal particles made from miniemulsion: a case study on testosterone and its structural analogues. Macromolecules. 2016;49(7): 2559-67.

53. Yáñez-Sedeño P, Campuzano S, Pingarrón JM. Electrochemical sensors based on magnetic molecularly imprinted polymers: a review. Anal Chim Acta. 2017;960:1-17.
54. Ben Aissa A, Herrera-Chacon A, Pupin RR, Sotomayor MDPT, Pividori MI. Magnetic molecularly imprinted polymer for the isolation and detection of biotin and biotinylated biomolecules. Biosens Bioelectron. 2017;88:101-8.

55. Descalzo AB, Somoza C, Moreno-Bondi MC, Orellana G. Luminescent core-shell imprinted nanoparticles engineered for targeted Förster resonance energy transfer-based sensing. Anal Chem. 2013;85(11):5316-20.

56. Mujahid A, Lieberzeit PA, Dickert FL. Chemical sensors based on molecularly imprinted sol-gel materials. Materials (Basel). 2010;3(4):2196-217.

57. Bakas I, Hayat A, Piletsky S, Piletska E, Chehimi MM, Noguer T, et al. Electrochemical impedimetric sensor based on molecularly imprinted polymers/sol-gel chemistry for methidathion organophosphorous insecticide recognition. Talanta. 2014;130:294-8.

58. Bates F, del Valle M. Voltammetric sensor for theophylline using sol-gel immobilized molecularly imprinted polymer particles. Microchim Acta. 2015;182(5-6):933-42.

59. Herrera-Chacon A, González-Calabuig A, Campos I, del Valle M. Bioelectronic tongue using MIP sensors for the resolution of volatile phenolic compounds. Sensors Actuators B Chem. 2018;258: 665-71.

60. Wang J, Musameh M, Lin Y. Solubilization of carbon nanotubes by Nafion toward the preparation of amperometric biosensors. J Am Chem Soc. 2003;125(9):2408-9.

61. Neto JDRM, Santos WDJR, Lima PR, Tanaka SMCN, Tanaka AA, Kubota LT. A hemin-based molecularly imprinted polymer (MIP) grafted onto a glassy carbon electrode as a selective sensor for 4-aminophenol amperometric. 2011;152(2):220-5.

62. Alizadeh T, Amjadi S. A tryptophan assay based on the glassy carbon electrode modified with a nano-sized tryptophan-imprinted polymer and multi-walled carbon nanotubes 2017;41(11):44934502.

63. Roushani M, Saedi Z, Hamdi F, Dizajdizi BZ. Preparation an electrochemical sensor for detection of manganese (II) ions using glassy carbon electrode modified with multi walled carbon nanotube-chitosan-ionic liquid nanocomposite decorated with ion imprinted polymer. J Electroanal Chem. 2017;804(September):1-6.

64. Matsuguchi M, Uno T. Molecular imprinting strategy for solvent molecules and its application for QCM-based VOC vapor sensing. Sensors Actuators B Chem. 2006;113(1):94-9.

65. Mazzotta E, Picca RA, Malitesta C, Piletsky SA, Piletska EV. Development of a sensor prepared by entrapment of MIP particles in electrosynthesised polymer films for electrochemical detection of ephedrine. Biosens Bioelectron. 2008;23(7):1152-6.

66. Ho KC, Yeh WM, Tung TS, Liao JY. Amperometric detection of morphine based on poly(3,4- ethylenedioxythiophene) immobilized molecularly imprinted polymer particles prepared by precipitation polymerization. Anal Chim Acta. 2005;542(1 SPEC. ISS):90-6.

67. Cetó X, Saint CP, Chow CWK, Voelcker NH, Prieto-Simón B. Electrochemical detection of $\mathrm{N}$-nitrosodimethylamine using a molecular imprinted polymer. Sensors Actuators B Chem. 2016;237: 613-20.

68. Gholivand MB, Torkashvand M. A novel high selective and sensitive metronidazole voltammetric sensor based on a molecularly imprinted polymer-carbon paste electrode. Talanta. 2011;84(3): 905-12.

69. Khan SI, Chillawar RR, Tadi KK, Motghare RV. Molecular imprinted polymer based impedimetric sensor for trace level determination of digoxin in biological and pharmaceutical samples. Curr Anal Chem. 14(5):474-82.

70. Mourad R, El badry Mohamed M, Frag EYZZ, El-Boraey HA, EL-Sanafery SS, El-Boraey HA, et al. A novel molecularlyimprinted potentiometric sensor for the fast 
determination of bisoprolol fumarate in biological samples. Electroanalysis. 2021;33(1):66-74.

71. Garcia-Cruz A, Ahmad OS, Alanazi K, Piletska E, Piletsky SA. Generic sensor platform based on electro-responsive molecularly imprinted polymer nanoparticles (e-NanoMIPs). Microsystems Nanoeng. 2020 Dec;6(1):83.

72. Dyer DJ. Photoinitiated synthesis of grafted polymers. In: Surfaceinitiated polymerization I. Berlin/Heidelberg: Springer-Verlag; 2006. p. 47-65.

73. Khlifi A, Gam-Derouich S, Jouini M, Kalfat R, Chehimi MM. Melamine-imprinted polymer grafts through surface photopolymerization initiated by aryl layers from diazonium salts. Food Control. 2013;31(2):379-86.

74. Kidakova A, Reut J, Boroznjak R, Öpik A, Syritski V. Advanced sensing materials based on molecularly imprinted polymers towards developing point-of-care diagnostics devices. Proc Est Acad Sci. 2019;68(2):158-67.

75. Özcan L, Şahin Y. Determination of paracetamol based on electropolymerized-molecularly imprinted polypyrrole modified pencil graphite electrode 2007;127(2):362-369.

76. Wang R, Wang L, Yan J, Luan D, Sun T, Wu J, et al. Rapid, sensitive and label-free detection of pathogenic bacteria using a bacteria-imprinted conducting polymer film-based electrochemical sensor. Talanta. 2021;226:122135.

77. Ma Y, Shen XL, Zeng Q, Wang LS. A glassy carbon electrode modified with graphene nanoplatelets, gold nanoparticles and chitosan, and coated with a molecularly imprinted polymer for highly sensitive determination of prostate specific antigen. Microchim Acta. 2017;184(11):4469-76.

78. Skoog DA, Holler FJ, Crouch SR. Principles of instrumental analysis. 7th ed. Cengage Learning: Boston, MA; 2018.

79. Barsoukov E, Macdonald JR, editors. Impedance Spectroscopy: Theory, Experiment, and Applications, Second Edition. John Wiley \& Sons, Ltd; 2005.

80. Florea A, Cristea C, Vocanson F, Səndulescu R, Jaffrezic-Renault $\mathrm{N}$. Electrochemical sensor for the detection of estradiol based on electropolymerized molecularly imprinted polythioaniline film with signal amplification using gold nanoparticles. Electrochem Commun. 2015;59:36-9.

81. Feier B, Blidar A, Pusta A, Carciuc P, Cristea C. Electrochemical sensor based on molecularly imprinted polymer for the detection of cefalexin. Biosensors. 2019;9(1).

82. Di Masi S, Garcia Cruz A, Canfarotta F, Cowen T, Marote P, Malitesta C, et al. Synthesis and application of ion-imprinted nanoparticles in electrochemical sensors for copper (II) determination. ChemNanoMat. 2019;5(6):754-60.

83. Kamel AH, Jiang X, Li P, Liang R. A paper-based potentiometric sensing platform based on molecularly imprinted nanobeads for determination of bisphenol A 2018;10(31):3890-3895.

84. da Silva JL, Buffon E, Beluomini MA, Pradela-Filho LA, Gouveia Araújo DA, Santos AL, et al. Non-enzymatic lactose molecularly imprinted sensor based on disposable graphite paper electrode. Anal Chim Acta. 2021;1143:53-64.

85. Guzmán-Vázquez De Prada A, Martínez-Ruiz P, Reviejo AJ, Pingarrón JM. Solid-phase molecularly imprinted on-line preconcentration and voltammetric determination of sulfamethazine in milk. Anal Chim Acta. 2005;539(1-2):125-32.

86. Hassan AHA, Sappia L, Moura SL, Ali FHM, Moselhy WA, Sotomayor M del PT, et al. Biomimetic magnetic sensor for electrochemical determination of scombrotoxin in fish. Talanta 2019;194(July 2018):997-1004.

87. Kamel AH, Moreira FTC, F. Sales MG. Biomimetic sensor potentiometric system for doxycycline antibiotic using a molecularly imprinted polymer as an artificial recognition element. Sens Lett. 2011;9(5):1654-60.
88. Lopes F, Pacheco JG, Rebelo P, Delerue-Matos C. Molecularly imprinted electrochemical sensor prepared on a screen printed carbon electrode for naloxone detection. Sensors Actuators B Chem. 2017;243:745-52.

89. Stojanovic Z, Erdőssy J, Keltai K, Scheller FW, Gyurcsányi RE. Electrosynthesized molecularly imprinted polyscopoletin nanofilms for human serum albumin detection. Anal Chim Acta. 2017;977:1-9.

90. Waffo AFT, Yesildag C, Caserta G, Katz S, Zebger I, Lensen MC, et al. Fully electrochemical MIP sensor for artemisinin. Sensors Actuators B Chem. 2018;275:163-73.

91. Ozcelikay G, Kurbanoglu S, Yarman A, Scheller FW, Ozkan SA. Au-Pt nanoparticles based molecularly imprinted nanosensor for electrochemical detection of the lipopeptide antibiotic drug Daptomycin. Sensors Actuators B Chem. 2020;320:128285.

92. Moura SL, Fajardo LM, Cunha L dos A, Sotomayor MDPT, Machado FBC, Ferrão LFA, et al. Theoretical and experimental study for the biomimetic recognition of levothyroxine hormone on magnetic molecularly imprinted polymer. Biosens Bioelectron 2018;107(November 2017):203-210.

93. Rapini R, Canfarotta F, Mazzotta E, Malitesta C, Marrazza G, Piletsky S, et al. NanoMIP-based approach for the suppression of interference signals in electrochemical sensors. Analyst. 2019;144(24):7290-5.

94. Raziq A, Kidakova A, Boroznjak R, Reut J, Öpik A, Syritski V. Development of a portable MIP-based electrochemical sensor for detection of SARS-CoV-2 antigen. Biosens Bioelectron. 2021;178:113029.

95. Canfarotta F, Czulak J, Guerreiro A, Cruz AG, Piletsky S, Bergdahl GE, et al. A novel capacitive sensor based on molecularly imprinted nanoparticles as recognition elements. Biosens Bioelectron. 2018;120(May):108-14.

96. Florea A, Cowen T, Piletsky S, De Wael K. Electrochemical sensing of cocaine in real samples based on electrodeposited biomimetic affinity ligands. Analyst. 2019;144(15):4639-46.

97. Couto RAS, Costa SS, Mounssef B, Pacheco JG, Fernandes E, Carvalho F, et al. Electrochemical sensing of ecstasy with electropolymerized molecularly imprinted poly(ophenylenediamine) polymer on the surface of disposable screenprinted carbon electrodes. Sensors Actuators B Chem. 2019;290(January):378-86.

98. Cetó X, O’Mahony AM, Wang J, Del Valle M. Simultaneous identification and quantification of nitro-containing explosives by advanced chemometric data treatment of cyclic voltammetry at screen-printed electrodes. Talanta. 2013;107:270-6.

99. Piletsky S, Ahmad OS, Cruz AG. Electrochemical Sensor [Internet]. UK; 2019. p. WO/2019/234417. Available from: https://patentscope.wipo.int/search/en/detail.jsf?docId= WO2019234417

100. Lowdon JW, Diliën H, Singla P, Peeters M, Cleij TJ, van Grinsven $\mathrm{B}$, et al. MIPs for commercial application in low-cost sensors and assays - an overview of the current status quo. Sensors Actuators B Chem. 2020;325:128973.

101. Otto M, Thomas JDR. Model studies on multiple channel analysis of free magnesium, calcium, sodium, and potassium at physiological concentration levels with ion-selective electrodes. Anal Chem. 1985;57(13):2647-2651.

102. Cetó X, Voelcker NH, Prieto-Simón B. Bioelectronic tongues: new trends and applications in water and food analysis. Biosens Bioelectron. 2016;79:608-26.

103. del Valle M. Bioelectronic tongues employing electrochemical biosensors. In: Matysik F-M, editor. Trends in bioelectroanalysis. Cham: Springer International Publishing; 2017. p. 143-202.

104. del Valle M. Electronic tongues employing electrochemical sensors. Electroanalysis. 2010;22(14):1539-55. 
105. Ni Y, Kokot S. Does chemometrics enhance the performance of electroanalysis? Anal Chim Acta. 2008;626(2):130-46.

106. Huynh TP, Kutner W. Molecularly imprinted polymers as recognition materials for electronic tongues. Biosens Bioelectron. 2015;74:856-64.

107. Bueno L, El-Sharif HF, Salles MO, Boehm RD, Narayan RJ, Paixão TRLC, et al. MIP-based electrochemical protein profiling. Sensors Actuators B Chem. 2014;204:88-95.

108. El-Sharif HF, Stevenson D, Reddy SM. MIP-based protein profiling: a method for interspecies discrimination. Sensors Actuators B Chem. 2017;241:33-9.
109. Nandy Chatterjee T, Banerjee Roy R, Tudu B, Pramanik P, Deka $\mathrm{H}$, Tamuly $\mathrm{P}$, et al. Detection of theaflavins in black tea using a molecular imprinted polyacrylamide-graphite nanocomposite electrode. Sensors Actuators B Chem. 2017;246:840-7.

Publisher's note Springer Nature remains neutral with regard to jurisdictional claims in published maps and institutional affiliations. 